\title{
Maintaining Integrity Under Stress: Envelope Stress Response Regulation of Pathogenesis in Gram-Negative Bacteria
}

\author{
Claire L. Hews ${ }^{1}$, Timothy Cho ${ }^{2}$, Gary Rowley ${ }^{1 *}$ and Tracy L. Raivio ${ }^{2 *}$ \\ ${ }^{1}$ School of Biological Sciences, University of East Anglia, Norwich, United Kingdom, ${ }^{2}$ Department of Biological Sciences, \\ University of Alberta, Edmonton, $A B$, Canada
}

OPEN ACCESS

Edited by:

Alain Charbit,

INSERM U1151 Institut Necker

Enfants Malades Centre de Médecine Moléculaire (INEM), France

Reviewed by:

Monique L. Van Hoek, George Mason University,

United States

Matthew Cabeen,

Oklahoma State University,

United States

*Correspondence:

Gary Rowley

g.rowley@uea.ac.uk

Tracy L. Raivio

traivio@ualberta.ca

Specialty section:

This article was submitted to Molecular Bacterial Pathogenesis,

a section of the journal

Frontiers in Cellular and Infection

Microbiology

Received: 04 June 2019

Accepted: 19 August 2019

Published: 04 September 2019

Citation:

Hews CL, Cho T, Rowley G and Raivio TL (2019) Maintaining Integrity

Under Stress: Envelope Stress

Response Regulation of Pathogenesis

in Gram-Negative Bacteria.

Front. Cell. Infect. Microbiol. 9:313.

doi: 10.3389/fcimb.2019.00313
The Gram-negative bacterial envelope is an essential interface between the intracellular and harsh extracellular environment. Envelope stress responses (ESRs) are crucial to the maintenance of this barrier and function to detect and respond to perturbations in the envelope, caused by environmental stresses. Pathogenic bacteria are exposed to an array of challenging and stressful conditions during their lifecycle and, in particular, during infection of a host. As such, maintenance of envelope homeostasis is essential to their ability to successfully cause infection. This review will discuss our current understanding of the $\sigma^{\mathrm{E}}$ - and $\mathrm{Cpx}$-regulated ESRs, with a specific focus on their role in the virulence of a number of model pathogens.

Keywords: Gram-negative bacteria, envelope stress, pathogenesis, sigmaE response, Cpx response

\section{INTRODUCTION}

Bacteria encounter numerous harsh and fluctuating environments during their lifecycle. This is of particular concern to pathogenic bacteria which must be able to tolerate the challenging conditions and immune response within a host. Detection of potentially harmful changes in the environment, or damage caused by such conditions, are inducers of bacterial stress responses. The ability of a bacterium to sense and withstand these environmental stresses is crucial to its survival, particularly within a host, and to establish and maintain infection. Within the host, a bacterium is threatened by a milieu of noxious stresses including temperature and $\mathrm{pH}$ changes, antimicrobial compounds, bile salts, oxidative and nitrosative stress, and nutrient starvation. In addition, successful colonization and infection of hosts requires careful regulation of a multitude of virulence factors, many of which are envelope-localized.

Consisting of an inner membrane (IM), periplasmic space (PP) containing a thin peptidoglycan (PG) layer and an outer membrane (OM), the Gram-negative bacterial envelope functions as the interface between the cell and the extracellular environment. It serves as the gate for import and export, barrier to harmful substances, protects against shifting environmental conditions and is the location of many essential metabolic processes. The envelope is first subjected to harsh environmental conditions and must be able to withstand the associated stresses. The ability to maintain envelope homeostasis and quickly repair any damage to ensure integrity is dependent on envelope stress responses (ESRs).

ESRs are able to perceive the presence of extracellular stress and the disruption of periplasmic homeostasis that can arise as a result (e.g., damage to proteins in the outer membrane or periplasm). The ESRs are comprised of a series of regulatory cascades, which have independent 
and overlapping stimuli, as well as regulon members. They are grouped into two component signal transduction (2CST) systems and RNA polymerase-associating alternative sigma factors. Much of our understanding of the mechanisms and regulons of the ESRs have arisen from extensive work in Escherichia coli. The best characterized ESRs are regulated through the $\sigma^{\mathrm{E}}$ pathway and the 2CST system CpxRA, central to the responses to outer membrane/periplasmic and inner membrane stress, respectively. In addition, other key ESRs include the regulator of capsule synthesis (Rcs) response, responding, in broad terms, to outer membrane and peptidoglycan stresses (Guo and Sun, 2017; Wall et al., 2018) and the phage shock protein (Psp) response, responding to disruptions at the IM (reviewed in Darwin, 2013; Flores-Kim and Darwin, 2016). Further 2CST systems exist, in addition to CpxAR, including ZraSR and BaeSR. Studies describe ZraSR as a zinc responsive system (Appia-Ayme et al., 2012; Petit-Härtlein et al., 2015), but this 2CST system has not been studied in depth and its precise physiological role remains to be determined. BaeSR on the other hand, responds to antibiotics/toxic compounds (Raffa and Raivio, 2002; Macritchie and Raivio, 2009; Appia-Ayme et al., 2011). Though not thought of as strictly responding to envelope stress, other regulatory systems such as the PhoPQ and EnvZ/OmpR two-component systems regulate processes that impact the ability of bacteria to adapt to envelope stress.

This review will summarize our current understanding of the $\sigma^{\mathrm{E}}$ and Cpx ESRs. While by no means the only ESRs relevant to bacterial pathogenesis, a large body of work has been conducted in characterizing the mechanism and function of these two ESRs in various Gram-negative bacteria. The aim of this review is to explain the basic mechanisms of these systems, as well as discuss their relevance to physiology and pathogenesis in several Gram-negative bacteria. In particular, we wish to highlight recent findings since this topic was last reviewed (Rowley et al., 2006; Raivio, 2014; Fang et al., 2016).

\section{ENVELOPE BIOGENESIS}

Being the barrier between the intracellular and extracellular environment, proper formation and maintenance of the envelope is essential to bacterial viability during all stages of a pathogen's lifecycle. Envelope biogenesis is a complex process which involves several machineries (Silhavy et al., 2010). Here we briefly describe those that transport, fold and assemble envelope proteins, since misfolding and/or mis-localization of such proteins has been directly linked to induction of both the $\sigma^{\mathrm{E}}$ and Cpx ESRs.

Proteins destined for all compartments of the envelope begin their life in the cytoplasm. As such, these proteins must be transported into or across the IM. Two different pathways exist for protein transport across the IM; the Sec translocase pathway and the twin arginine translocase (Tat) pathway. The vast majority of secreted proteins utilize the Sec pathway (reviewed in Kuhn et al., 2017; Tsirigotaki et al., 2017), while the Tat system (reviewed in Lee et al., 2006; Palmer and Berks, 2012) transports proteins which have already been folded in the cytoplasm and those which also contain metal cofactors. Briefly, an unfolded protein is targeted to the Sec translocase machinery through the recognition of an $\mathrm{N}$-terminal signal peptide. SecYEG forms the IM channel through which unfolded proteins are transported. Transport across the IM is energized by the ATPase SecA. Proteins that are inserted into the IM can be inserted by SecYEG itself or utilize the IM protein YidC to assist in insertion. Once secreted across the IM, proteins can be modified and translocated in several different ways. A number of factors including the IM protein insertase YidC, together with proteases and associated regulators, play roles in the biogenesis and quality control of IM proteins (Akiyama, 2009; Luirink et al., 2012).

Lipoproteins are a special class of envelope proteins that are acylated at their $\mathrm{N}$-terminus and can be localized to either the IM or the OM (reviewed in Okuda and Tokuda, 2011; Szewczyk and Collet, 2016; Narita and Tokuda, 2017). In brief, after being secreted across the IM, lipoproteins undergo multiple modification steps that result in the addition of several acyl chains to an $\mathrm{N}$-terminal cysteine residue. OM-destined lipoproteins are trafficked to the OM by the Lol pathway. An IM complex consisting of the ATP-binding cassette (ABC) transporter LolCDE recognizes OM-destined lipoproteins based on the presence of an aspartate residue at the +2 residue and energizes the transfer of these proteins to a periplasmic chaperone LolA. LolA transports OM lipoproteins to the OM, where LolB, itself an OM lipoprotein, receives and inserts OM lipoproteins into the OM. As OM lipoproteins have been implicated in promoting the virulence of some Gram-negative organisms, the Lol pathway presents a potential target for the development of new therapies. For example, the surface-exposed lipoproteins of Neisseria spp. play roles in pathogenesis, such as immune evasion (reviewed in Hooda et al., 2017). More broadly, lipoproteins make up key members of envelope biogenesis machineries, such as the BAM complex (see below), and can indirectly impact many envelopelocalized virulence determinants.

Outer membrane biogenesis has been reviewed extensively elsewhere (Ruiz et al., 2006; Bos et al., 2007; Plummer and Fleming, 2016; Konovalova et al., 2017) therefore it will only be briefly described in this review for relevance to understand the contribution of the ESR to this process. All unfolded outer membrane proteins (uOMPs) are translocated via the Sec system. Once the uOMP reaches the periplasm, the signal sequence is cleaved and the nascent OMP is delivered by periplasmic chaperones (SurA and Skp) to the $\beta$-barrel assembly machinery (BAM) complex for folding and insertion into the outer membrane.

The BAM complex consists of five major components: BamA (YaeT), BamB (YfgL), BamC (NlpB), BamD (YfiO), and BamE (SmpA) (Plummer and Fleming, 2016). The genes encoding the BAM complex components are all regulated by $\sigma^{\mathrm{E}}$ (Skovierova et al., 2006; Lewis et al., 2008) and are discussed below. BamA, a member of the Omp85 protein family and originally identified in Neisseria meningitidis, is highly conserved across Gramnegative bacteria and is essential to the complex and bacterial viability (Voulhoux et al., 2003). Interestingly, while BamA is essential across all Gram-negative bacteria, the essentiality of the other four lipoprotein components varies; for example, BamD is essential for complex function in E. coli yet is not 
essential in Salmonella (Fardini et al., 2009). BamA formation is dependent on BamD with depletions in this lipoprotein resulting in BamA misfolding (Misra et al., 2015). In addition, BamB and BamD have been shown to bind unfolded BamA and assist in its localization and insertion into the outer membrane (Hagan et al., 2013).

Aside from its essentiality, there are numerous links between the BAM complex and pathogenicity. Salmonella bamE mutants are attenuated in mice (Lewis et al., 2008) and Salmonella Enteritidis $b a m B$ and $b a m D$ deletions render the pathogen less virulent with reduced expression of flagella and the type III secretion system (T3SS) (Amy et al., 2004; Fardini et al., 2007, 2009). In Yersinia enterocolitica, a bamB mutant is attenuated in mice with a significant reduction in the spleen bacterial burden after 3 days (Weirich et al., 2017). Additionally, a bamE deletion leads to OMP defects and rifampicin sensitivity in E. coli (Sklar et al., 2007a).

Recently, a number of studies have identified small molecule inhibitors of components of the BAM complex. Hagan et al. (2015) identified a 15-mer peptide fragment of BamA that binds to BamD. Since BamA formation is dependent on BamD and BamA is required for cell viability, substrate competition between the small molecule and the BamA substrate resulted in growth defects and increased sensitivity to vancomycin and rifampicin. BamA has been identified as a viable target for therapeutics with the development of a monoclonal antibody (MAB1) which binds to the extracellular loop of BamA and affects OM integrity (Storek et al., 2018). Furthermore, inhibition of Pseudomonas BamA by bacteriocins has also been observed, specifically lectin like bacteriocins (LlpAs). Bacteria often secrete these toxic compounds in order to selectively kill related bacteria and Ghequire et al. (2018) identified that LlpA resistant Pseudomonas carried mutations in the extracellular loop of BamA.

Put together, studies such as these support the consensus that understanding the process of OM biogenesis and its maintenance may provide new therapeutic routes against these pathogens.

\section{THE EXTRACYTOPLASMIC SIGMA FACTOR $\sigma^{\mathrm{E}}$}

Maintenance of the outer membrane depends largely on the extracytoplasmic sigma factor $\sigma^{\mathrm{E}}$ ( $r p o E$ ) (Rouvière et al., 1995). This ESR senses misfolded OMPs within the outer membrane and periplasmic space. Activation of the $\sigma^{\mathrm{E}}$ pathway involves a series of proteolytic cleavage events (Figure 1). Inducers of the $\sigma^{\mathrm{E}}$ pathway are summarized in Rowley et al. (2006) and include: oxidative stress, heat shock, carbon starvation and biofilm formation. More recently, acid stress (Muller et al., 2009) ultraviolet A (UVA) radiation, P22 phage and hypo-osmotic
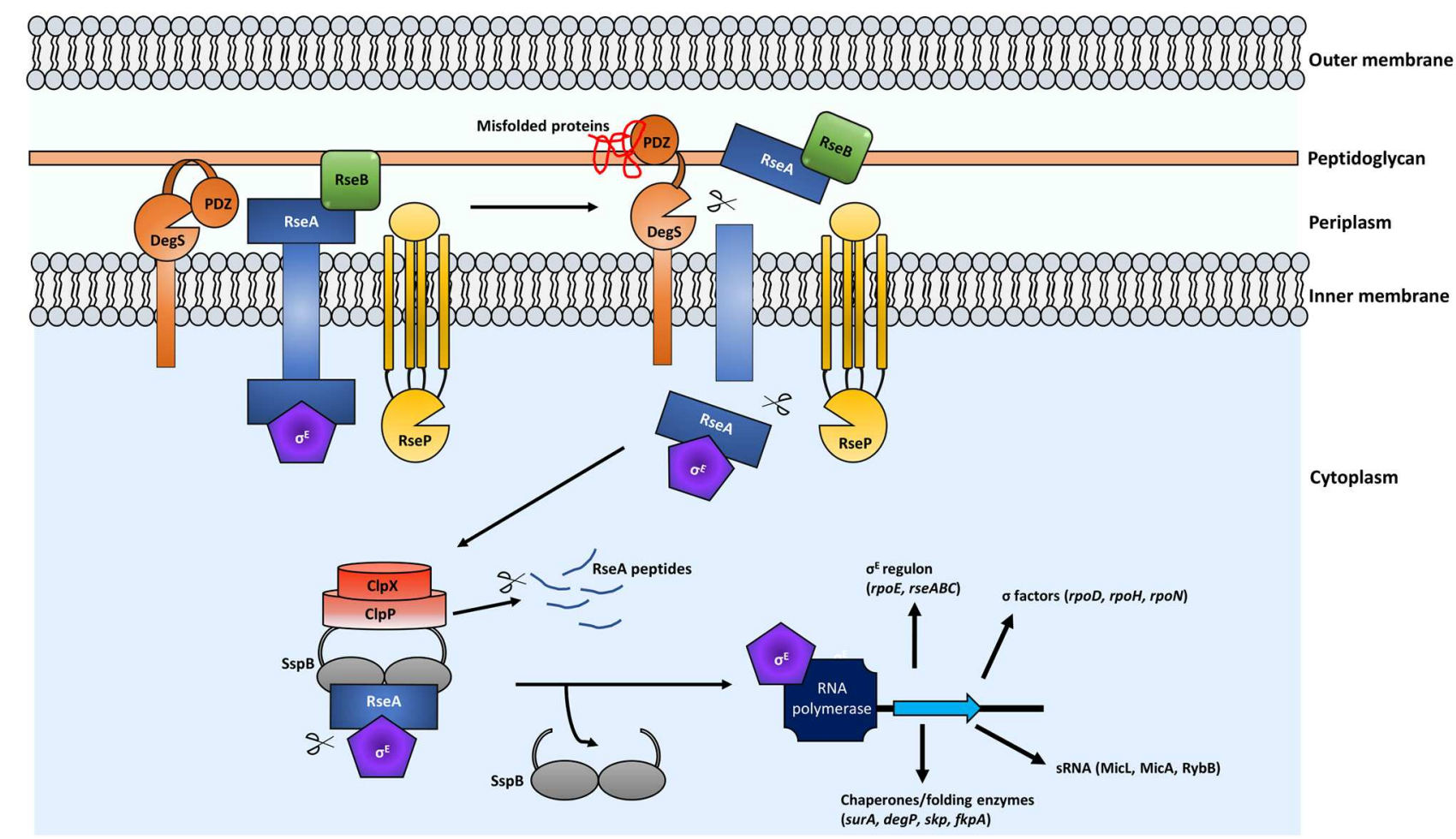

FIGURE 1 | Regulated intramembrane proteolysis (RIP) leads to activation of the $\sigma^{E}$ ESR. In the presence of misfolded proteins, a conformational change as a result of misfolded proteins binding to the PDZ domain of DegS occurs. Subsequently the protease domain of DegS is exposed and the periplasmic domain of RseA is cleaved. RseP then cleaves RseA at the cytoplasmic domain, releasing the $\sigma^{\mathrm{E}}$-bound RseA portion into the cytoplasm. Binding of the adaptor protein SspB to RseA- $\sigma^{E}$ recruits the ClpXP protease for degradation of RseA and release of free $\sigma^{\mathrm{E}} . \sigma^{\mathrm{E}}$ binds to RNA polymerase and transcription of the $\sigma^{\mathrm{E}}$ regulon is induced. 
shock have also been shown to induce the $\sigma^{\mathrm{E}}$ ESR (Amar et al., 2018). Generally, activation of the $\sigma^{\mathrm{E}}$ pathway is due to accumulation of misfolded and/or mis-translocated OMPs or LPS within the periplasm (Rowley et al., 2006). In laboratory E. coli strains and Yersinia enterocolitica, rpoE is an essential gene (De Las Peñas et al., 1997a; Heusipp et al., 2003), although suppressor mutations do enable rpoE mutants to be viable (De Las Peñas et al., 1997a). Insertion mutations in $y d c Q$, a putative DNA binding protein (Button et al., 2007), and two genes of unknown function $y h b W$ and $p t s N$ (Hayden and Ades, 2008) have been identified to enable the $r p o E$ deletion in $E$. coli to be tolerated. Numerous studies have shown that $r p o E$ is not essential in other bacteria including Salmonella (Humphreys et al., 1999; Skovierova et al., 2006), although it does make a major contribution to their virulence. Interestingly, it has recently been reported that loss of the LPS O-antigen renders an rpoE deletion lethal in Salmonella (Amar et al., 2018). The authors propose that when present, the LPS O-antigen provides protection to the Salmonella OM allowing an rpoE deletion, and presumably the OM defects that result, to be tolerated.

Encoded within the $r p o E$ operon are the $\sigma^{\mathrm{E}}$ inhibitors $r s e A$ and $r s e B$ (Dartigalongue et al., 2001). Activation of the $\sigma^{\mathrm{E}}$ ESR occurs via regulated intramembrane proteolysis (RIP) and begins at the transmembrane protein RseA (Figure 1). Under normal physiological conditions, RseA is bound to $\sigma^{\mathrm{E}}$ and as such, sequesters it from interacting with RNA polymerase to influence gene expression. RseB is also a negative regulator of the $\sigma^{\mathrm{E}}$ pathway and when bound to RseA it increases affinity of this complex for $\sigma^{\mathrm{E}}$, resulting in inhibition of the pathway (De Las Peñas et al., 1997b; Collinet et al., 2000; Ahuja et al., 2009; Chaba et al., 2011) (Figure 1).

In the presence of misfolded proteins, the $\sigma^{\mathrm{E}}$ cascade begins with the cytoplasmic cleavage of RseA by the protease DegS (Ades et al., 1999; Alba et al., 2002; Kanehara et al., 2002; Li et al., 2009). The DegS site-1 protease (S1P) is a transmembrane protein embedded within the IM (Figure 1). The presence of uOMPs in the periplasm induces conformational changes in DegS, via interaction between the C-terminus of the uOMPs with the PDZ protease domain of DegS. This conformational change exposes the protease region for RseA cleavage (Walsh et al., 2003; Sohn et al., 2009). DegS is essential in E. coli laboratory strains however construction of a degS mutant has been reported in certain extraintestinal E. coli strains, Salmonella Typhimurium (Rowley et al., 2005), and Vibrio cholerae (Mathur et al., 2007). Although viable, $S$. Typhimurium $\operatorname{deg} S$ mutants are defective in their ability to colonize the host and cause infection (Redford et al., 2003) while $V$. cholerae degS mutants are unable to activate the $\sigma^{\mathrm{E}}$ cascade following antimicrobial peptide (AMP) treatment (Mathur et al., 2007). Interestingly, degS mutants can still mount a $\sigma^{\mathrm{E}}$ response to certain stresses (Rowley et al., 2005). In $S$. Typhimurium, $\sigma^{\mathrm{E}}$ can be activated by acid stress independently of DegS and misfolded proteins (Muller et al., 2009).

Following cleavage by DegS, the RseP (YaeL) protease is recruited and the cytoplasmic portion of RseA, bound to $\sigma^{\mathrm{E}}$, is released into the cytoplasm (Alba et al., 2002; Kanehara et al., 2002) (Figure 1). RseP is a site-2 protease (S2P), therefore, its cleavage of RseA can only occur after cleavage by the S1P. Li et al. (2009) identified that S1P cleavage leads to S2P cleavage due to the exposure of a hydrophobic amino acid (Val-148) at the carboxyl-terminus of RseA. Inhibition of RseP prevents cleavage of RseA and results in a lethal sequestration of $\sigma^{\mathrm{E}}$. Interestingly, a sRNA, RseX, was shown to reduce the levels of OMPs OmpA and OmpC when overexpressed and this led to survival of rseP mutant cells in E. coli (Douchin et al., 2006). Recently, Konovalova et al. (2018) identified a eukaryotic matrix metalloprotease (MMP) inhibitor which targets RseP and leads to toxic uOMP accumulation. This was found to be due to loss of $\sigma^{\mathrm{E}}$-regulated sRNAs, MicA and RybB, which play an important role in downregulating expression of uOMPs (see Figure 2 and described in detail below).

Following release of RseA bound $\sigma^{\mathrm{E}}$, the adaptor SspB binds to the complex and recruits the ATP-dependent ClpXP protease (Figure 1) (Flynn et al., 2004; Baker and Sauer, 2012). $\mathrm{ClpX}$ is an AAA+ ATPase and ClpP is a peptidase (Baker and Sauer, 2012) and in the final stage of the $\sigma^{\mathrm{E}}$ cascade the protein complex releases $\sigma^{\mathrm{E}}$ from RseA (Chaba et al., 2007). Analysis of the roles of the ClpXP protease provides evidence that individual components of the $\sigma^{\mathrm{E}}$ pathway play important roles in bacterial pathogenesis with Salmonella clpX and $c l p P$ mutants being attenuated in macrophages and BALB/c mice (Yamamoto et al., 2001).

Once released from RseA, free $\sigma^{\mathrm{E}}$ binds RNA polymerase as a cofactor, subsequently inducing expression of the $\sigma^{\mathrm{E}}$ regulon (Figure 1). The $\sigma^{\mathrm{E}}$ regulon has been linked with virulence in a number of pathogens (discussed below). The function of proteins that form part of the $\sigma^{\mathrm{E}}$ regulon across many bacterial species include those for DNA repair, metabolism, OM biogenesis and chaperones and proteases required for maintaining periplasmic homeostasis (Dartigalongue et al., 2001; Rhodius et al., 2006; Skovierova et al., 2006).

$\sigma^{\mathrm{E}}$-regulated chaperones and proteases are of importance to $\mathrm{OM}$ biogenesis and the tolerance of extracytoplasmic stress particularly during host infection (see below). The major $\sigma^{\mathrm{E}}$ regulated chaperones are Skp, SurA, FkpA, and HtrA (DegP). The function of these proteins has been studied extensively in E. coli, however they are well-conserved across multiple Gram-negative species including E. coli, Salmonella, and Yersinia sp.

SurA possesses peptidyl-prolyl cis/trans isomerase (PPIase) activity which folds proteins via catalysis of the rate-limiting cis/trans isomerization of peptidyl bonds around proline residues (bacterial PPIases are reviewed in Ünal and Steinert, 2014). In addition to, and independently of, its PPIase activity SurA also functions as a periplasmic chaperone (Behrens et al., 2001). A major role of the periplasmic chaperones is to prevent aggregation of uOMPs; however, SurA and a further chaperone Skp, encoded within the bamA genomic region, have been shown to play a role in uOMP folding in cooperation with the BAM complex. Studies in E. coli have shown that SurA interacts with BamA in order to deliver uOMPs (Sklar et al., 2007b; Vuong et al., 2008), while Skp assists in the folding of OmpA and SkpOmpA complexes interact with BamA (Patel and Kleinschmidt, 2013). Interestingly, double deletion mutants of $s k p$ and $s u r A$ result in a lethal phenotype and as a result, it is understood that they function in two different but overlapping pathways of 


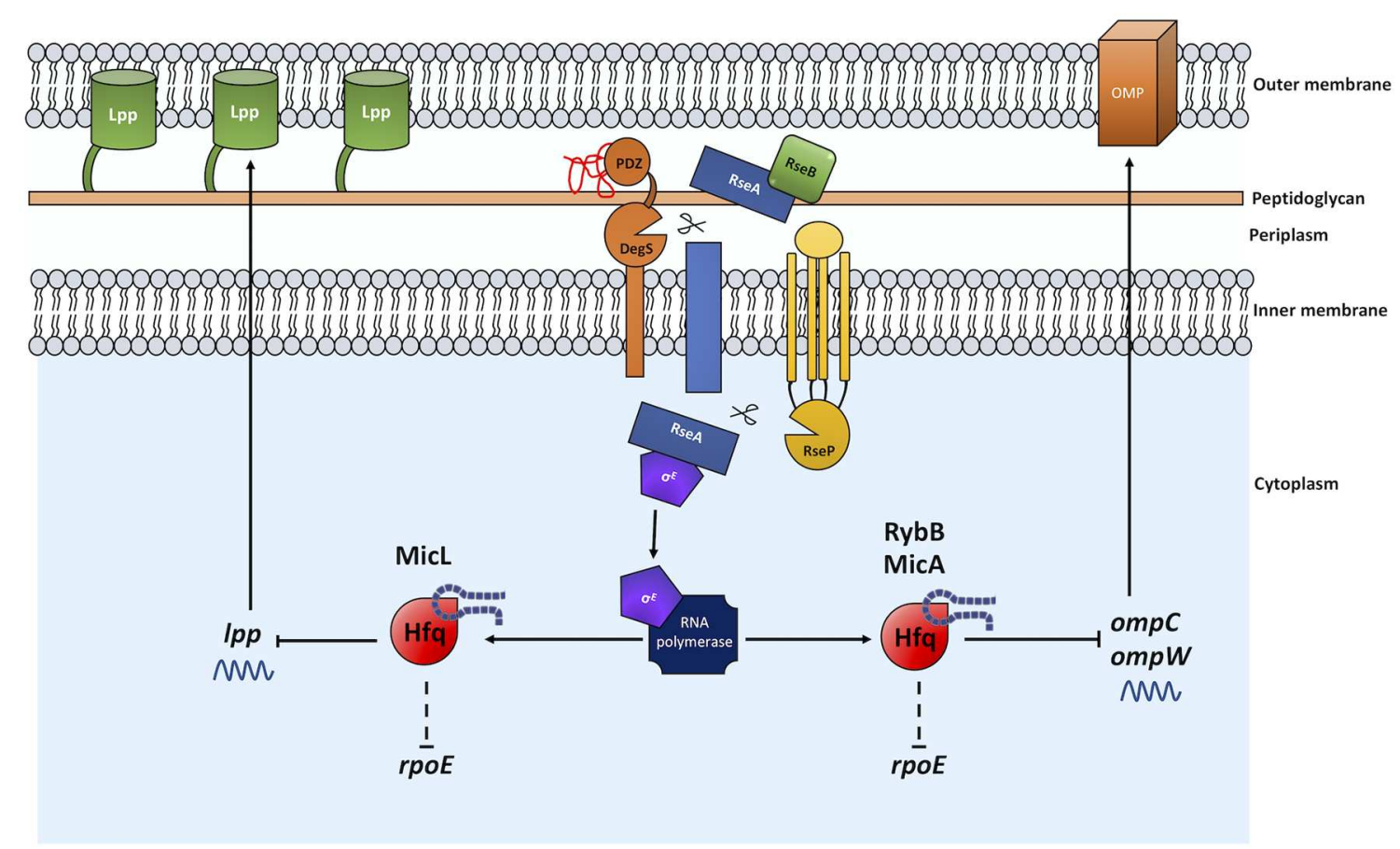

FIGURE 2 | The sRNAs MicL, RybB, and MicA exert control over OM homeostasis and the $\sigma^{\mathrm{E}}$ envelope stress response. The presence of misfolded OMPs activates the $\sigma^{\mathrm{E}}$ ESR through a proteolytic cleavage cascade as described in Figure 1. The $\sigma^{\mathrm{E}}$ regulon includes the Hfq-associating sRNAs micL, rybB, and micA. MicL inhibits Ipp mRNA translation leading to a reduction in Lpp production. RybB and MicA reduce ompC and ompW mRNA levels, reducing the flux of uOMPs into the periplasm during stress and $\sigma^{\mathrm{E}}$ inducing conditions. Normal arrows represent positive regulation and blunt arrows indicate negative regulation. Dashed lines indicate where regulation is indirect and/or where intermediate factors are unknown.

OM biogenesis (Sklar et al., 2007b). Despite this, overexpression of $f k p A$ can compensate for the lethal phenotype and enables Salmonella growth at $37^{\circ} \mathrm{C}$ (Ge et al., 2014). Like SurA, FkpA is a PPIase and chaperone and its chaperone activity is independent of PPIase activity. In addition to a role in OM biogenesis, overexpression of FkpA was shown to rescue $\operatorname{degP}(h t r A)$ mutants during heat shock (Arie et al., 2001). DegP is unique in its ability to function as both a chaperone and a protease and this switch is dependent on temperature. At low temperatures, DegP is a chaperone transporting misfolded and unfolded OMPs; however, at higher temperatures it possesses protease function degrading proteins that are beyond repair (Spiess et al., 1999).

In addition to chaperones and proteases, encoded within the $r p o E$ regulon are a number of sRNAs with regulatory functions. sRNAs bind to specific mRNA targets and subsequently activate or repress the mRNA through affecting its stability or by inhibiting its translation (Holmqvist and Wagner, 2017). sRNAs have been shown to regulate the $\sigma^{\mathrm{E}}$, CpxRA, and Rcs ESRs (reviewed in Frohlich and Gottesman, 2018). The sRNAs regulated by $\sigma^{\mathrm{E}}$ are MicA (SraD), RybB and MicL (RyeF/SlrA) and they function to regulate the $\sigma^{\mathrm{E}}$ response and expression of a group of OMPs and lipoproteins (Figure 2). Ultimately, the sRNAs regulated by $\sigma^{\mathrm{E}}$ downregulate other processes, such as uOMP production, that may otherwise lead to continued activation of the ESR. All $\sigma^{\mathrm{E}}$-regulated sRNAs associate with the RNA chaperone $\mathrm{Hfq}$ and inactivation of $\mathrm{Hfq}$ is an activator of the $\sigma^{\mathrm{E}}$ ESR (Figueroa-Bossi et al.,
2006; Sittka et al., 2007; Klein and Raina, 2015). This Hfqdependent regulation does indeed warrant further study in order to better understand the underlying mechanisms. In Salmonella, loss of $\mathrm{Hfq}$ has been shown to result in an increase in DegS-dependent cleavage of RseA, and this is likely due to increased accumulation of uOMPs (FigueroaBossi et al., 2006). In addition, Guisbert et al. (2007) identified that downregulation of the mRNA of eight OMPs (ts $x$, fiu, $o m p X, o m p A, o m p F, l p p, o m p C, y h c N)$ is dependent on Hfq. Therefore, through association with sRNAs that function to downregulate $\mathrm{UOMP}$ production (described in more detail below) and control the $\sigma^{\mathrm{E}}$ response, $\mathrm{Hfq}$ can indirectly inactivate $\sigma^{\mathrm{E}}$.

MicL is an $80 \mathrm{nt}$ transcript, processed from a primary transcript of $308 \mathrm{nt}$, and is located within the cutC gene. Also referred to as SlrA (suppressing lap RNA), MicL can function as a multicopy suppressor of lapA lapB mutations (the Lap proteins are essential in the process of LPS synthesis and for cell viability) (Klein et al., 2014). To date, the only known mRNA target of MicL is lpp (Guo et al., 2014). The lpp gene encodes an OM lipoprotein, commonly referred to as Lpp or Braun's lipoprotein (Braun, 1975), which is covalently attached to the peptidoglycan layer. Lpp is the most abundant protein in E. coli and functions to stabilize the cell envelope through its OM-peptidoglycan interaction (for recent review, see Asmar and Collet, 2018). MicL targets $l_{p p}$ by preventing translation of its mRNA, thereby inhibiting Lpp protein production (Guo et al., 2014). This could 
be seen as counterproductive; why would $\sigma^{\mathrm{E}}$, a mechanism in place to maintain $\mathrm{OM}$ integrity, prevent production of a cell envelope stabilizing protein? Studies indicate that in doing so, demand on the Lol lipoprotein assembly machinery is lessened. This, in turn, enables increased production of the BamD lipoprotein and LPS assembly components (primarily LptE), thus increasing the folding of uOMPs and LPS production in the periplasm, which would otherwise further induce $\sigma^{\mathrm{E}}$ (Guo et al., 2014).

The remaining sRNAs MicA and RybB were first identified in $E$. coli as $\sim 70 \mathrm{nt}$ and $\sim 80 \mathrm{nt}$, respectively. Since, these sRNAs have also been identified and shown to be conserved in Salmonella (Papenfort et al., 2006). These sRNAs overlap in function and downregulate expression of outer membrane porins in an $\mathrm{Hfq}$-dependent manner. MicA is responsible for the decrease in ompA mRNA, particularly during stationary phase when $\sigma^{\mathrm{E}}$ is highly active (Vogel and Papenfort, 2006). RybB has been shown to specifically decrease ompC and $o m p W$ mRNA levels via Hfq (Johansen et al., 2006). As a result, these sRNAs contribute to the maintenance of envelope homeostasis via downregulation of OMP production, in a similar fashion to MicL, reducing transport of uOMPs into the periplasm during conditions of stress when the periplasmic uOMP content may already be elevated.

Interestingly, MicA negatively regulates the response regulator PhoP of the PhoPQ 2CST via base-pairing within the phoP translation initiation site (Coornaert et al., 2010). The contribution of the PhoPQ 2CST system to OM remodeling in Salmonella has been well-documented (Ernst et al., 2001; Dalebroux and Miller, 2014; Dalebroux et al., 2014) and crosstalk between this system and the $\sigma^{\mathrm{E}}$ ESR provides further evidence that different ESR pathways overlap. This connection emphasizes the necessity of a coordination of responses that modify and maintain the envelope during infection and bacterial stress. The negative regulation of the PhoPQ 2CST system by $\sigma^{\mathrm{E}}$ demonstrates that under some ESR-inducing conditions, it may be detrimental to the bacterium for certain ESRs to be simultaneously active.

\section{$\sigma^{\mathrm{E}}$ and Bacterial Pathogenesis Salmonella}

Salmonella is an intracellular pathogen capable of causing infection in both humans and animals. Serovars of Salmonella sp. can reside in different niches and vary in the type of infection they cause. S. Typhimurium and $S$. Enteritidis are both examples of enteric bacteria typically causing food poisoning, while $S$. Typhi is responsible for the more serious and systemic Typhoid fever.

A large number of $\sigma^{\mathrm{E}}$-regulated proteins, chaperones and PPIases have been implicated in Salmonella virulence (Figure 3A). During infection, Salmonella resides within macrophages in the Salmonella containing vacuole (SCV), a site of ROS production and associated stress. It has been shown previously that $\sigma^{\mathrm{E}}$ is important for survival within macrophages and in a murine infection model (Humphreys et al., 1999). rpoE is upregulated in macrophages (Eriksson et al., 2003) and the $\sigma^{\mathrm{E}}$ regulon is required for Salmonella resistance to oxidative stress (Testerman et al., 2002; Li et al., 2015a).
Furthermore, in the invasive serovar $S$. Typhi, responsible for the systemic infection Typhoid fever, $r p o E$ mutants are attenuated for invasion and intracellular survival. In addition, expression of the pathogenicity islands SPI-1 and SPI-2, encoding the T3SSs required for invasion and intracellular survival, are reduced (Xie et al., 2016; Zhang et al., 2016). $\sigma^{\mathrm{E}}$ also plays an important role in the downregulation of cellular processes. The anti-FlhC ${ }_{2} \mathrm{D}_{4}$ complex factor $\mathrm{RflP}$ (YdiV) is activated by $\sigma^{\mathrm{E}}$ and RflP functions to target the $\mathrm{FlhC}_{2} \mathrm{D}_{4}$ master regulator of flagellar synthesis to the ClpXP protease for degradation in S. Typhimurium (Spöring et al., 2018). As such, in this non-typhoidal serovar, $\sigma^{\mathrm{E}}$ is involved in the downregulation of Salmonella motility. Ultimately, research suggests that downregulation of flagellar synthesis can aid in host immune evasion, thus increasing bacterial fitness during infection. Conversely, in $S$. Typhi, $\sigma^{\mathrm{E}}$ has been found to promote flagellar gene expression, during osmotic stress, via upregulation of fliA (Du et al., 2011). The authors of this study propose that RpoE may respond to the hyperosmotic environment in the intestinal lumen and increase motility to enable $S$. Typhi invasion of epithelial M cells. These interesting discrepancies indicate that $\sigma^{\mathrm{E}}$ may be involved in differences between localized and systemic Salmonella infections. Much of the groundwork in understanding the role of $\sigma^{\mathrm{E}}$ in Salmonella pathogenesis has been performed in S. Typhimurium; however, these data indicate the continuing need to expand these studies into other serovars, especially those which cause invasive and systemic disease.

Further links between $\sigma^{\mathrm{E}}$ and coordination of virulence gene expression have also been shown: the SPI-2 pathogenicity island is, in part, regulated by the SsrAB 2CST system and an $S$. Typhimurium $r p o E$ mutant has reduced expression of the SsrB-dependent secreted effector protein SseB (Osborne and Coombes, 2009). As described above, encoded within the bamA region is the $\sigma^{\mathrm{E}}$-regulated periplasmic chaperone, $s k p$. Rowley et al. (2011) identified significant attenuation of a $s k p$ mutant in a murine infection model. Additionally, $f k p A$ mutants of Salmonella Copenhagen show reduced levels of intracellular survival (Horne et al., 1997), although studies in $S$. Typhimurium demonstrate that $f k p A$ mutants are unaffected for intracellular survival, unless also combined with a deletion in surA or $\operatorname{deg} P$ (Humphreys et al., 2003). Deletion of surA leads to loss of adhesion and invasion of epithelial cells by $S$. Typhimurium (Sydenham et al., 2000). In addition, the surA mutant was identified as an attenuated live vaccine. Sydenham et al. (2000) showed that when mice were challenged with WT $S$. Typhimurium, following a challenge with the surA mutant, the WT was unable to colonize the host. These findings certainly point toward an important role for $\sigma^{\mathrm{E}}$-regulated periplasmic chaperones in infection and also for the identification of new therapeutic targets.

In addition to the proteins regulated by $\sigma^{\mathrm{E}}$, the sRNAs have also been linked to virulence. MicA and RybB are both upregulated inside macrophages (Srikumar et al., 2015). Furthermore, MicA has a role in biofilm formation in Salmonella and this was understood to be due to MicA targeting of ompA and phoP mRNAs (Kint et al., 2010). 


\section{A Salmonella enterica}

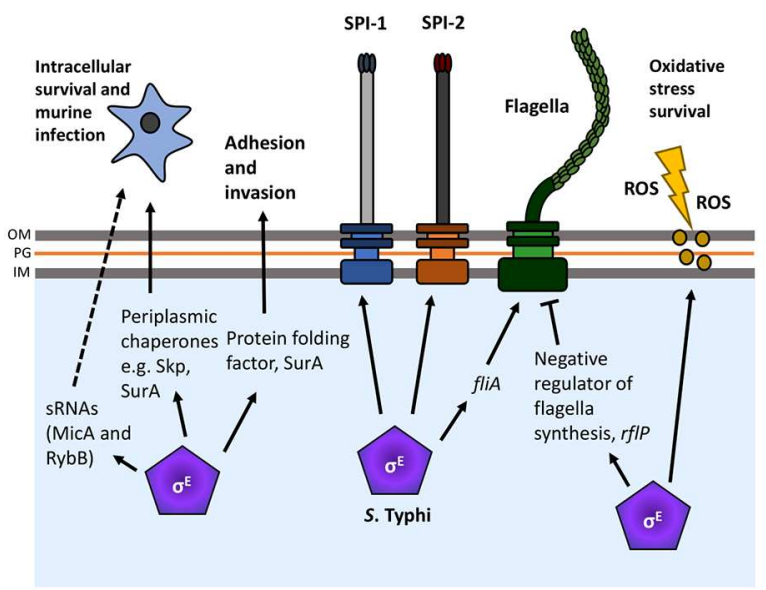

\section{B Escherichia coli}

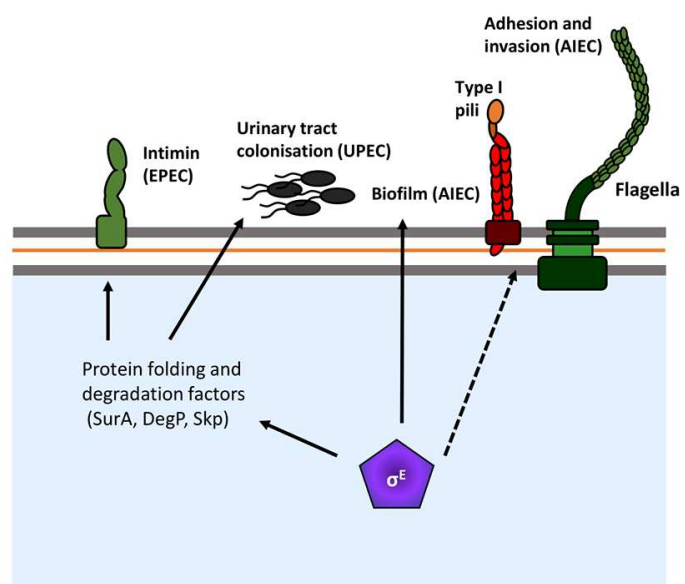

\section{c Pseudomonas sp.}

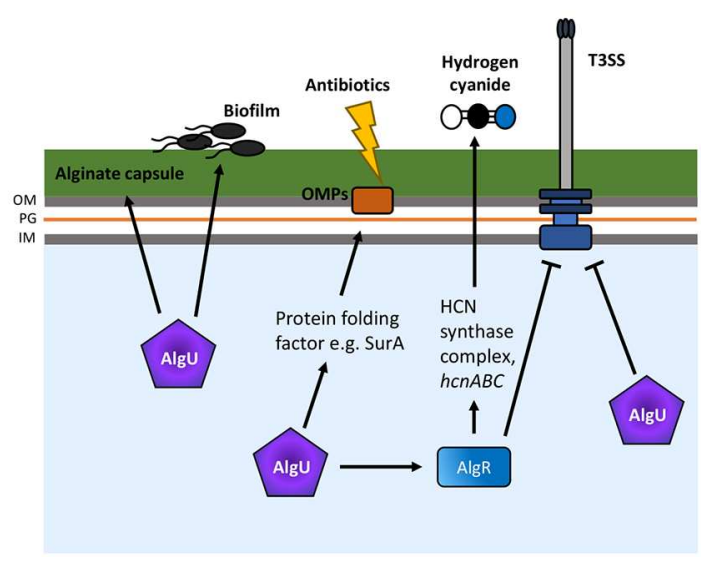

FIGURE $3 \mid \sigma^{E}$-regulation of virulence factors in Escherichia coli, Salmonella enterica, and Pseudomonas spp. The $\sigma^{E}$ ESR regulates many virulence factors and surface structures, such as adhesins, in multiple bacteria such as Salmonella enterica (A), E. coli (B), and Pseudomonas spp. (C), primarily via the upregulation of periplasmic folding factors. Additionally, a number of bacteria-specific factors are regulated by this ESR. These include hydrogen cyanide in Pseudomonas sp. and the Salmonella pathogenicity islands (SPIs), SPI-1, and SPI-2. Normal arrows represent positive regulation and blunt arrows indicate negative regulation. Dashed lines indicate where regulation is indirect and/or where intermediate factors are unknown.

Aside from a role in pathogenicity and intracellular survival, $r p o E$ is also required for Salmonella resistance to antimicrobial peptides (AMPs) during infection. Specifically, rpoE mutants were shown to be reduced in survival when challenged with the bactericidal/permeability-increasing protein (BPI)-derived peptide P2 and the murine $\alpha$-defensin cryptdin-4 (Crp4), both of which are known to disrupt the cell envelope (Crouch et al., 2005).

Studies identifying the role of previously uncharacterized $\sigma^{\mathrm{E}}$-regulated genes are continually emerging. Recently, Morris et al. (2018) demonstrated that the $\sigma^{\mathrm{E}}$-regulated lipoprotein YraP contributes to $\mathrm{OM}$ integrity and the ability to cause murine infection. Results showed that infection of mice with a yraP mutant resulted in significant attenuation, particularly in the liver, compared to WT. However, the yraP mutant strain was not attenuated for adhesion, invasion or intracellular survival in J774, Caco-2 or primary bone marrow-derived macrophages. As such, the precise mechanism behind the attenuation of $\triangle y r a P$ in mice is yet to be elucidated.

\section{Escherichia coli}

The role of $\sigma^{\mathrm{E}}$ in the pathogenesis of $E$. coli has not been studied to the same extent as in Salmonella, potentially because $r p o E$ is an essential gene in laboratory E. coli. However, a small number of studies have demonstrated a role for $\sigma^{\mathrm{E}}$-regulated chaperones in the virulence of different pathogenic E. coli strains (Figure 3B).

Extraintestinal E. coli strains are capable of colonizing and causing infection outside of their usual intestinal niche. Uropathogenic E. coli (UPEC) is one such example and is a major 
cause of urinary tract infections (UTIs). It has been described that the $\sigma^{\mathrm{E}}$-regulated chaperones $\operatorname{deg} P$ and $s k p$ are important for E. coli urinary tract colonization (Redford and Welch, 2006). Enteropathogenic E. coli (EPEC) is a major cause of infant diarrhea and its ability to adhere to intestinal enterocytes is central to its pathogenesis (Walsham et al., 2016). The adhesin intimin is required for attachment to epithelial cells and it has been shown that the $\sigma^{\mathrm{E}}$-regulated chaperones SurA, DegP and Skp are needed for the insertion of intimin into the OM (Bodelon et al., 2009).

Another E. coli pathovar, adherent-invasive Escherichia coli (AIEC), is capable of invading intestinal epithelial cells (Yang et al., 2017). $\sigma^{\mathrm{E}}$ is required for biofilm formation of Crohn's disease associated AIEC; inhibition of $\sigma^{\mathrm{E}}$ by overexpression of RseAB resulted in a significant reduction of AIEC biofilm formation (Chassaing and Darfeuille-Michaud, 2013). In addition, $\sigma^{\mathrm{E}}$ is important for AIEC adhesion and invasion of epithelial cells (Rolhion et al., 2007; Chassaing and Darfeuille-Michaud, 2013). Studies demonstrate that inhibition of $\sigma^{\mathrm{E}}$ leads to a reduction in adhesion and invasion and that this is in part, due to reduced expression of flagella and type 1 pili. However, the exact mechanisms behind the observed phenotypes are yet to be fully elucidated (Chassaing and Darfeuille-Michaud, 2013). In contrast to $\sigma^{\mathrm{E}}$, other stress responses including the 2CST system CpxAR have been implicated in E. coli virulence to a greater extent and this will be described in detail below.

\section{Pseudomonas}

Pseudomonas aeruginosa is an opportunistic pathogen associated with high morbidity and mortality in patients with underlying respiratory disease such as cystic fibrosis (Gellatly and Hancock, 2013). P. aeruginosa strains exist in mucoid or non-mucoid forms but generally environmental strains and those which lead to initial colonization of the host are non-mucoid in nature (Rao et al., 2011). It has been demonstrated that the conversion to the mucoid form occurs during host colonization and it appears that the conditions, particularly in the CF lung, positively select for mucoid $P$. aeruginosa. As expected, mucoid strains are most commonly associated with CF patients; however, they have also been isolated from non-CF patients (Govan and Deretic, 1996).

The conversion from non-mucoid to mucoid is mediated by mutations in $m u c A$. The $m u c A$ gene forms part of the $\operatorname{alg} U$, $m u c A, m u c B, m u c C$ operon which is homologous to the $r p o E$ rseA rseB rseC operon in E. coli (Rowley et al., 2006). Normally, MucA sequesters AlgU activity (as does RseA for RpoE); however, mutations in $m u c A$, typically mucA22, result in constitutive algU activation (Mathee et al., 1999). The algU regulator, sharing $79 \%$ amino acid sequence homology with E. coli $\sigma^{\mathrm{E}}$ (Potvin et al., 2008), controls expression of the alginate biosynthesis operon, formed of 12 genes for biosynthesis and export which starts at $\operatorname{alg} D$. Induction of the AlgU pathway results in production of the exopolysaccharide alginate and is of particular importance for bacterial protection and persistence in the lung (Figure 3C).

Activation of the AlgU pathway occurs via RIP (reviewed in Damron and Goldberg, 2012), as does activation of the $\sigma^{\mathrm{E}}$ pathway. In addition, Pseudomonas has functional equivalents of the E. coli proteases DegP, DegS and RseP; MucD, AlgW, and MucP, respectively (Pandey et al., 2016). Further to this, the ClpXP proteases are conserved in Pseudomonas and are required to release AlgU from MucA in the cytoplasm (Qiu et al., 2008).

Yu et al. (1995) showed that Pseudomonas algU mutants can be complemented with E. coli rpoE, and this complementation provided the alg $U$ mutant with resistance to paraquat-induced ROS stress. It has not yet been shown whether the lethal $r p o E$ deletion in $E$. coli can be rescued by $\operatorname{alg} U$.

Environmental stress has been shown to induce mutations in mucA. Mathee et al. (1999) described that when a nonmucoid strain, PA01, was grown in a biofilm treated with hydrogen peroxide, mucoid variants with mutations in $m u c A$ formed. The authors propose that these findings suggest antioxidant therapy may be a therapeutic option for patients, as this may aid in preventing the switch from non-mucoid to mucoid.

In addition to regulating alginate production, $\mathrm{Alg} \mathrm{U}$ regulates genes required for virulence (Figure 3C). Interestingly, an algU deletion increases systemic virulence (Yu et al., 1996), hence it appears that $\operatorname{alg} U$ is required for infection in the respiratory but not systemic environment. The ability to form a biofilm is a major virulence factor of many bacterial pathogens and $\mathrm{AlgU}$ is important for biofilm formation, specifically in non-mucoid strains (Bazire et al., 2010). AlgR is a component of the 2CST system AlgZR and is regulated by AlgU (Okkotsu et al., 2014). $\mathrm{AlgR}$, in turn, regulates hydrogen cyanide ( $\mathrm{HCN})$ production which studies indicate is an important Pseudomonas virulence factor. Produced under low oxygen conditions, $\mathrm{HCN}$ has been shown to be responsible for killing in the Caenorhabditis elegans infection model (Gallagher and Manoil, 2001). Additionally, both $\mathrm{AlgR}$ and $\mathrm{AlgU}$ have been shown to suppresses the Pseudomonas T3SS in mucoid strains (Okkotsu et al., 2014).

Several of the chaperones and proteases involved in maintenance of $\mathrm{OM}$ and periplasmic homeostasis in E. coli and Salmonella are conserved in Pseudomonas sp. A recent paper described a surA deletion re-sensitizes a MDR strain to antibiotics, suggesting that SurA could be a promising therapeutic target (Klein et al., 2019). Moreover, in support of this conclusion, deletion of surA also increased sensitivity to normal human serum.

\section{Conclusions and Further Evidence of Roles of $\sigma^{\mathrm{E}}$ in Bacterial Virulence}

The $\sigma^{\mathrm{E}}$ ESR has been implicated in other bacterial species in addition to those described above. Unfortunately, due to limitations in space, a detailed discussion is beyond the scope of this particular review. In brief, additional examples of a role for $\sigma^{\mathrm{E}}$ in pathogenesis include work in Vibrio cholerae and Yersinia sp. In Vibrio cholerae, an rpoE deletion resulted in a highly attenuated strain that was unable to colonize the intestine (Kovacikova and Skorupski, 2002). Moreover, a $\sigma^{\mathrm{E}}$-regulated sRNA, VrrA, inhibits production of OmpA and subsequently inhibits outer membrane vesicle (OMV) formation (Song and Wai, 2009). VrrA was also shown to control biofilm formation through translational repression of $r b m C$, a $V$. cholerae specific biofilm matrix protein. When VrrA was overexpressed, biofilm levels were reduced and, as such, VrrA may assist in the 


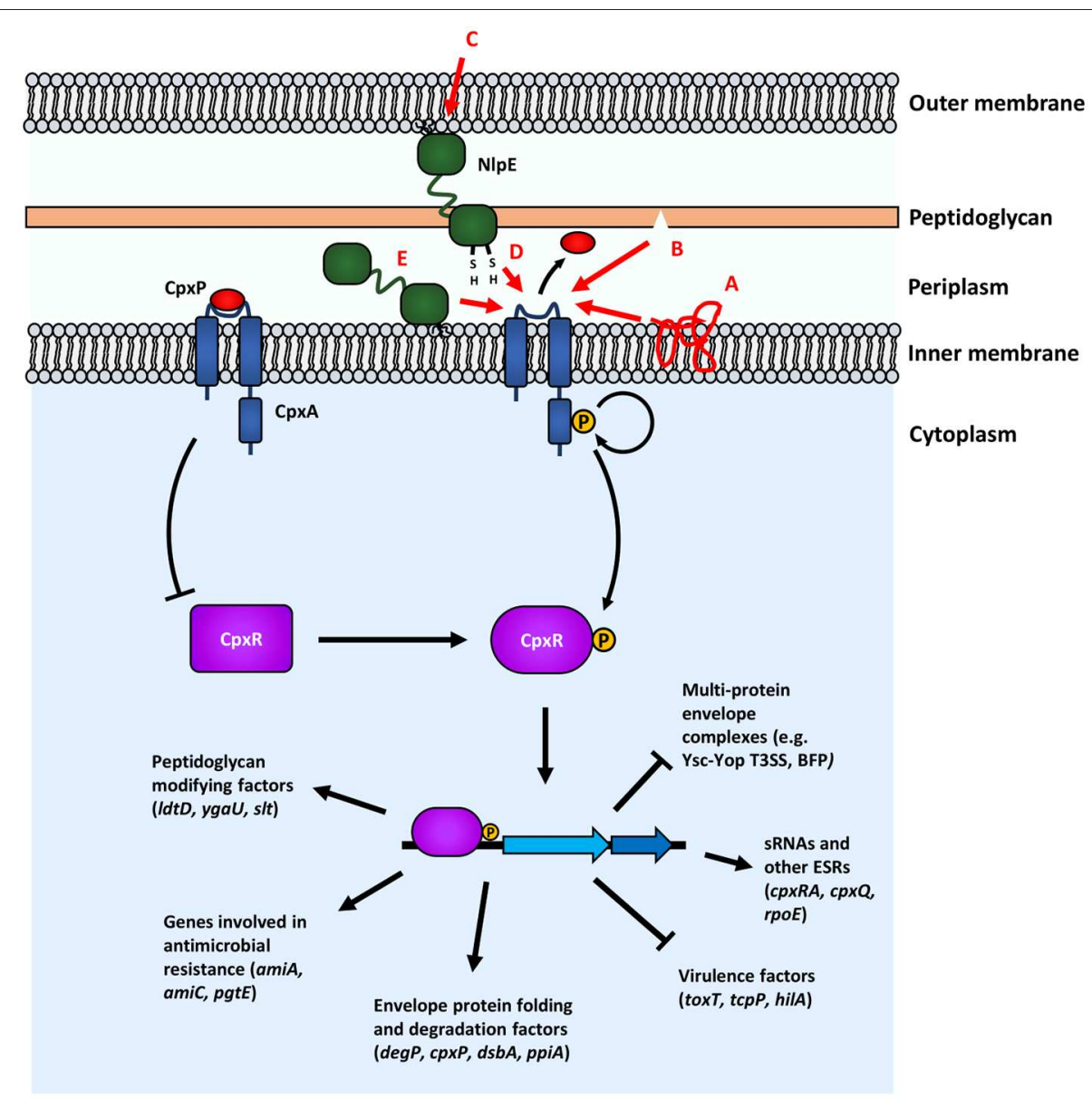

FIGURE 4 | The Cpx response senses and responds to a variety of envelope stresses. Proposed inducing cues of the Cpx response are shown with red arrows and corresponding red lettering: (A) stresses that lead to protein misfolding; (B) damage to peptidoglycan; (C) surface signals; (D) monitoring periplasmic redox states; (E) OM lipoprotein trafficking defects. In the absence of induction, the phosphatase activity of CpxA keeps CpxR unphphosphorylated. Upon induction, phosphotransfer from phosphorylated CpxA to CpxR leads to CpxR-P modulation of transcription of genes involved in several processes. Genes shown are representative, but not exhaustive of the Cpx regulon.

transition of $V$. cholerae from attachment in the intestine to shedding and uptake by new hosts (Song et al., 2014). In Yersinia sp. the $\sigma^{\mathrm{E}}$-regulated chaperone SurA is required for $Y$. pseudotuberculosis adhesion to HeLa cells (Obi and Francis, 2013) and a $Y$. pestis surA mutant is attenuated in mice (Southern et al., 2016). The vast array of studies highlighted in this review demonstrate that $\sigma^{\mathrm{E}}$ is of extreme importance to not only survival of Gram-negative bacteria, through the maintenance of the extracytoplasmic OM, but also to the ability to cause such successful and varied infections.

\section{THE CpXRA TWO-COMPONENT SYSTEM}

The Cpx (conjugative pilus expression) response is a widelyconserved ESR in Gram-negative bacteria. As a canonical 2CST system, signaling in the Cpx response occurs through the sensor histidine kinase (SHK) CpxA and the response regulator CpxR (Figure 4). Under non-inducing conditions, the phosphatase activity of CpxA keeps CpxR in an unphosphorylated state
(Raivio and Silhavy, 1997). Under inducing conditions, CpxA autophosphorylates and transfers the phosphate group to CpxR. One of the earliest findings implicating the Cpx response in a concrete role in responding to envelope stress was the finding that activated alleles of $c p x A$ suppress the toxicity of the IMlocalized fusion protein LamB-LacZ-PhoA (Cosma et al., 1995). Since then, much work has been done to characterize the function of the Cpx response in mitigating envelope stress (which is more comprehensively reviewed in Vogt and Raivio, 2012; Raivio, 2014). A variety of inducing cues activate the Cpx response, many, although not all, of which are thought to affect the integrity of the envelope and/or protein-folding outside of the cytoplasm. These include alkaline $\mathrm{pH}$ (Danese and Silhavy, 1998), aberrant expression of the Pap pilus (Jones et al., 1997), adhesion to hydrophobic surfaces (Otto and Silhavy, 2002), antimicrobial peptides (Audrain et al., 2013), and copper (Yamamoto and Ishihama, 2006). Some of the best characterized members of the Cpx regulon are genes that are involved in envelope protein folding and degradation, further suggesting a role for the 
Cpx response in maintaining the integrity of the envelope by monitoring and responding to stress due to misfolded envelope proteins. These Cpx-regulated genes include those encoding the periplasmic chaperones CpxP and Spy (Danese and Silhavy, 1998; Raivio et al., 2000), the chaperone/protease DegP/HtrA (Danese et al., 1995), the disulfide bond-forming oxidoreductase DsbA (Pogliano et al., 1997), peptidyl-prolyl isomerase PpiA (Pogliano et al., 1997), IM protease HtpX and YccA, a factor that modulates IM proteolytic activity (Shimohata et al., 2002; Yamamoto and Ishihama, 2006; Raivio et al., 2013). The Cpx response also appears to regulate other ESRs, such as the operon encoding the regulatory components of the $\sigma^{\mathrm{E}}$ response $(r p o E-r s e A B C)$, which is negatively regulated (Price and Raivio, 2009). It is not clear why the $\sigma^{\mathrm{E}}$ response is negatively regulated by the Cpx response, given that both the Cpx and $\sigma^{\mathrm{E}}$ responses respond to stress related to protein misfolding in the envelope. Future studies investigating this cross-regulation may shed more light onto the specific role of these ESRs and the purpose of this regulatory antagonism.

Although ostensibly a "two-component" system, other regulatory factors, such as the periplasmic protein $\mathrm{CpxP}$ and the OM lipoprotein NlpE are known to regulate signaling in some capacity in the Cpx response. Overexpression of NlpE is a wellknown inducer of the Cpx response in E. coli (Danese et al., 1995; Snyder et al., 1995; Danese and Silhavy, 1998). However, NlpE is not essential in signaling all, indeed most, Cpx-inducing cues, such as PapG overexpression (DiGiuseppe and Silhavy, 2003). The crystal structure of NlpE was solved by Hirano et al. (2007) and was shown to possess two distinct $\beta$-barrel domains at its $\mathrm{N}$ - and C-terminus connected by an unstructured flexible linker. Comparisons of NlpE homologs in Gram-negative organisms reveal that there are two broad types of NlpE: type I (possessing the full-length protein) and type II (lacking the C-terminal domain) (Hirano et al., 2007).

One of the roles that NlpE is thought to play is as a signaler of adhesion to hydrophobic abiotic surfaces, as well as host cell surfaces (Otto and Silhavy, 2002; Shimizu et al., 2016). The structure of NlpE and its implication in sensing surface signals suggest that during signaling, conformational changes in NlpE could lead to the C-terminus "reaching down" and interacting with the sensing domain of CpxA (Hirano et al., 2007). However, the precise mechanism of NlpE signaling to the Cpx response remains largely unknown, although recent papers, that will be discussed shortly, present new insights into the function of NlpE. Interestingly, multi-drug resistant (MDR) strains of Acinetobacter baumannii were found to express increased levels of NlpE and this was implicated in higher biofilm formation on abiotic surfaces, despite the fact that the A. baumannii NlpE is type II (i.e., lacks a C-terminal domain) (Siroy et al., 2006).

Studies in both the non-pathogenic K-12 strain MC4100 and enterohemorrhagic E. coli (EHEC) have suggested NlpE functions as a sensor of surface adhesion both to hydrophobic glass surfaces (K-12, EHEC) and undifferentiated Caco-2 cells (EHEC) (Otto and Silhavy, 2002; Shimizu et al., 2016). Sensing surface adhesion is thought to be an important initial step in the ability of a bacteria to colonize both biotic and abiotic surfaces, particularly as a biofilm (reviewed in Belas, 2014). In line with this, surface sensing by NlpE was implicated by Shimizu et al. (2016) as an important regulatory event in promoting virulence factor expression in EHEC. However, the role of the Cpx response in surface sensing was recently disputed by Kimkes and Heinemann (2018) who did not find induction of Cpxregulated yebE- or $c p x P$-GFP fluorescent reporters in response to adhesion to hydrophobic glass in a microfluidics setup nor when they attempted to recreate the original experimental conditions of Otto and Silhavy (2002). However, a close examination of these studies reveals a number of methodological differences that make it difficult to make a conclusive verdict as to whether or not NlpE is involved in surface sensing. Clearly, these conflicting conclusions point to a need for further study clarifying the role of NlpE in surface adhesion.

Recent studies have pointed to other potential roles for NlpE, specifically, as a sensor for monitoring stress related to lipoprotein trafficking and periplasmic redox state (Grabowicz and Silhavy, 2017; Delhaye et al., 2019). Deleting lolB, which encodes for the OM receptor lipoprotein responsible for inserting lipoproteins trafficked to the OM, has a severe deleterious effect on cell growth presumably because essential OM lipoproteins are not trafficked properly (Tanaka et al., 2001; Grabowicz and Silhavy, 2017). Deleting $c p x R$ or $n l p E$ in genetic backgrounds that suppress this toxicity, restores toxicity (Grabowicz and Silhavy, 2017). Conversely, activating CpxA is able to restore growth in the deleterious lolB null background suggesting that the Cpx response, through $\mathrm{NlpE}$, is able to sense lipoprotein trafficking defects and mitigate this stress (Grabowicz and Silhavy, 2017). This makes sense, as NlpE is itself an OM lipoprotein and its own trafficking would be affected by lipoprotein trafficking defects. This model is further supported by work showing that the $\mathrm{N}$ terminal domain of NlpE, which would be physically close to CpxA at the IM in the absence of trafficking to the OM, physically interacts with and is able to activate CpxA (Delhaye et al., 2019). Furthermore, it is known that forcing NlpE to localize to the IM induces the Cpx response (Miyadai et al., 2004; Delhaye et al., 2016). Future studies should work to characterize what Cpx regulon members are responsible for mitigating stress related to lipoprotein mistrafficking. Grabowicz and Silhavy (2017) raised the intriguing possibility that in the absence of the canonical Lol trafficking pathway, an alternative, Cpx-regulated pathway might exist to traffick lipoproteins, as essential OM lipoproteins such as BamD are still trafficked to the $\mathrm{OM}$ in the absence of LolB during Cpx-activation.

In addition to this novel role as a sensor for lipoprotein trafficking, NlpE may also act as a sensor for stress related to protein folding. Disulfide bonds in periplasmic proteins are introduced by DsbA, the absence of which leads to Cpx activation (Bardwell et al., 1991; Delhaye et al., 2019). This activation is dependent on the presence of NlpE and NlpE lacking C-terminal cysteine residues (and therefore without its normal disulfide bond) activates the response, suggesting that the function the of the C-terminal domain of NlpE is at least partly as an indicator of periplasmic redox state (Delhaye et al., 2019). It should be noted that the C-terminal cysteine residues of NlpE are conserved across Gram-negative bacteria that possess type I NlpE, suggesting that this function of NlpE may be important in several different organisms (Hirano et al., 2007). 
These studies present a model in which NlpE acts as a "Swiss army knife," with distinct domains involved in sensing distinct stresses and signaling to CpxA to maintain the integrity of the envelope. Although not directly regulating virulence in most cases, these functions of NlpE have potential ramifications on pathogenesis of bacteria at various stages of infection. The transition from the environment to the host may increase oxidative stress or alter periplasmic redox status, necessitating a means to monitor and respond to these changes. A bacterial cell must "know" that it is in contact with an appropriate surface to express virulence factors or form biofilms, making surface sensing a key initial step during infection. Lipoproteins play a key role in the biogenesis of many envelope components, including virulence factors such as secretion machineries, and as such, monitoring lipoprotein trafficking is important for their proper expression. Overall, further studies of the signaling functions of NlpE may reveal important insights into the role that the Cpx response plays as a whole during infection.

CpxP is a periplasmic protein that bears structural homology to the Cpx-regulated periplasmic chaperone Spy (Kwon et al., 2010). The role of CpxP in signaling is thought to be primarily negative. $c p x P$ is one of the most highly expressed members of the Cpx regulon upon activation but overexpression of CpxP inhibits activation of CpxA (Raivio et al., 1999, 2013; DiGiuseppe and Silhavy, 2003). This inhibition likely occurs through direct interaction with the periplasmic sensing domain of CpxA (Raivio et al., 1999, 2000; Zhou et al., 2011; Tschauner et al., 2014). It is thought that CpxP, in the presence of misfolded envelope proteins, will be titrated away from CpxA, and subsequently degraded by DegP/HtrA (Buelow and Raivio, 2005; Isaac et al., 2005; Tschauner et al., 2014). However, the role of CpxP in Cpx signaling is, like NlpE, not essential for most studied inducing cues, as inducers such as NlpE overexpression and alkaline $\mathrm{pH}$ do not require $\mathrm{CpxP}$ to activate the response (DiGiuseppe and Silhavy, 2003). Interestingly, although induction via alkaline $\mathrm{pH}$ doesn't require $\mathrm{CpxP}, \operatorname{cpxP}$ mutants are hypersensitized to alkaline pH (Danese and Silhavy, 1998; DiGiuseppe and Silhavy, 2003). Overall, NlpE and CpxP, under the conditions in which they have been studied, rather than acting as integral players in signal transduction, appear to allow for finer regulation of the response, both as a damper of activation, as in the case of CpxP or as an enabler for other sensory inputs, as in the case of NlpE.

Interestingly, recent work has revealed the existence of a conserved, RNase E and Hfq-dependent sRNA encoded in the 3' untranslated region (UTR) of the $c p x P$ mRNA, aptly named CpxQ (Chao and Vogel, 2016). In Salmonella, CpxQ targeted several envelope proteins, including the sodium-proton antiporter NhaB, the major subunit of the type I pilus FimA, and the periplasmic chaperone protein Skp (Chao and Vogel, 2016). CpxQ was needed for optimal survival in the presence of carbonyl cyanide 3-chlorophenylhydrazone (CCCP), a reagent that disrupts the proton motive force (PMF) at the IM (Chao and Vogel, 2016). This, combined with the regulation of NhaB by CpxQ suggests that its role is connected to preserving the $\mathrm{PMF}$ at the IM. In E. coli, CpxQ was shown to repress CpxP production by decreasing translation of $c p x P$ mRNA (Grabowicz et al., 2016). Furthermore, CpxQ was shown to help combat stress due to a mutant allele of the OM protein $\operatorname{LamB}[\operatorname{lamB}(A 23 D)]$, that aberrantly tethers it to the IM, a lethality that is suppressed by Cpx activation (Cosma et al., 1995; Grabowicz et al., 2016). Interestingly, it was not the regulation of CpxP by CpxQ but repression of the periplasmic chaperone Skp that was responsible for alleviating the toxicity of $\operatorname{LamB}(A 23 \mathrm{D})$ (Grabowicz et al., 2016). Skp is thought to facilitate the aberrant insertion of LamB(A23D) into the IM, creating a pore and disrupting the PMF at the IM, a stress that is relieved by the repression of Skp by CpxQ (Grabowicz et al., 2016). This is consistent with the finding that CpxQ is involved in alleviating stress due to CCCP (Chao and Vogel, 2016).

Overall, these results point to a growing body of evidence for the role of sRNAs in mediating the stress-alleviating effects of ESRs. It had previously been shown that the Cpx response regulates the expression of several sRNAs, such as cyaR, omrA, $o m r B$, and $r p r A$ and that these sRNA are involved in a regulatory network that not only regulates CpxRA but also links it to other 2CST systems such as EnvZ/OmpR (Raivio et al., 2013; Vogt et al., 2014). In the $\sigma^{\mathrm{E}}$ response, $\sigma^{\mathrm{E}}$-regulated sRNAs can directly target and repress porins and OM lipoproteins that are potential sources of envelope stress (Gogol et al., 2011; Guo et al., 2014). Given the demonstrated relevance of sRNAs to envelope stress adaptation, further study of CpxQ presents an opportunity to better understand the mechanism of how the Cpx response alleviates envelope stress.

\section{The Cpx Response and Bacterial Pathogenesis Escherichia coli}

The relevance of the Cpx response to the physiology and pathogenesis of various strains of Escherichia coli is welldocumented (Figure 5A). Studies in MP1, a commensal strain of $E$. coli isolated from mice, found that deleting $c p x R$ caused a severe colonization defect in mice (Lasaro et al., 2014). Further, cpxRA deleted strains of UPEC are attenuated in colonization and virulence in both mouse and zebrafish models (Debnath et al., 2013).

At the molecular level, multiple studies have focused on envelope-localized protein machineries such as the UPEC P-pilus and the enteropathogenic E. coli (EPEC) type IV bundle-forming pilus (BFP), which are assembled at the envelope and play key roles in adhesion to host cells (Wullt et al., 2000; Tobe and Sasakawa, 2001). Overexpressing PapE and PapG subunits of the $\mathrm{P}$ pilus activates the Cpx response but the activation of the Cpx response is not due to non-specific protein misfolding stress but rather the presence of a specific N-terminal extension on PapE involved in pilus subunit polymerization, suggesting that the Cpx response specifically monitors pilus assembly (Jones et al., 1997; Lee et al., 2004). In support of this, in the absence of $c p x R, \mathrm{~K}-12$ strains containing $\mathrm{P}$ pili genes produce shorter $\mathrm{P}$ pili, suggesting that the Cpx response is needed for efficient pilus expression and assembly (Hung et al., 2001). CpxR is also able to repress the expression of the pap pilin genes directly (Hernday et al., 2004). Similar roles for the Cpx response in both efficient assembly and inhibition of the elaboration of pili have been demonstrated in 


\section{A Escherichia coli}

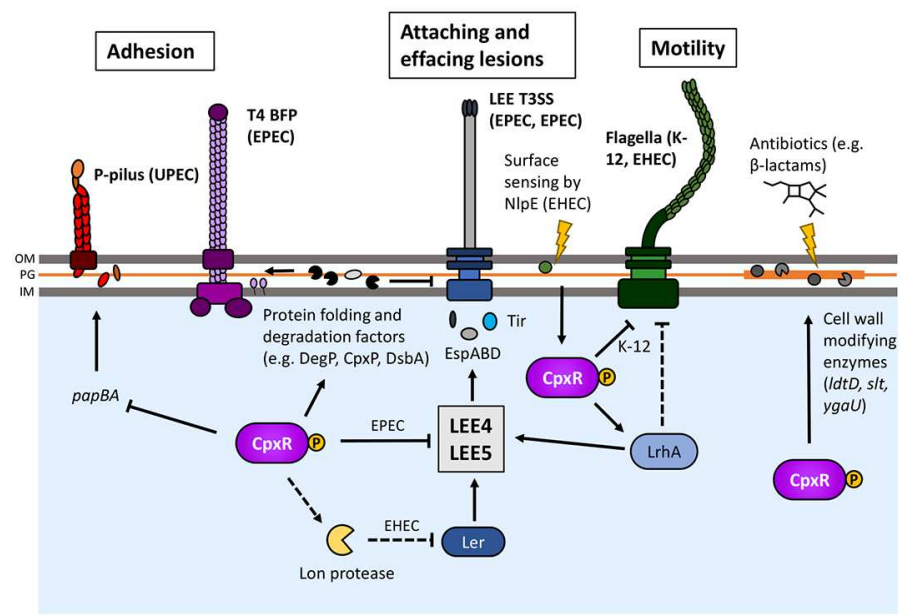

\section{c Vibrio cholerae}

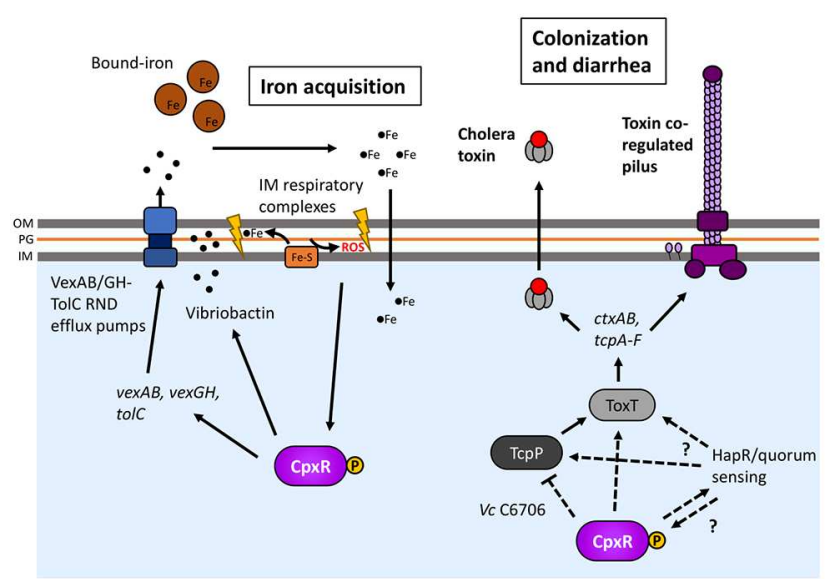

\section{B Salmonella enterica}

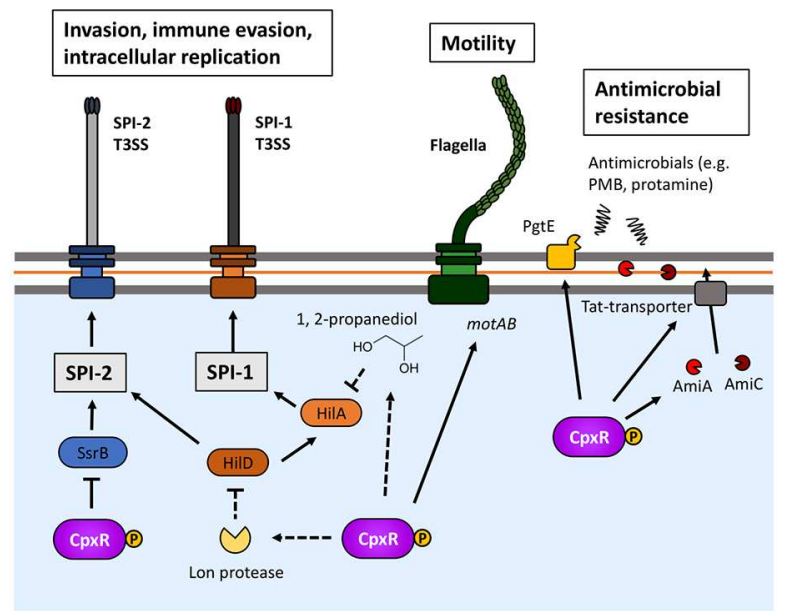

D Yersinia spp.

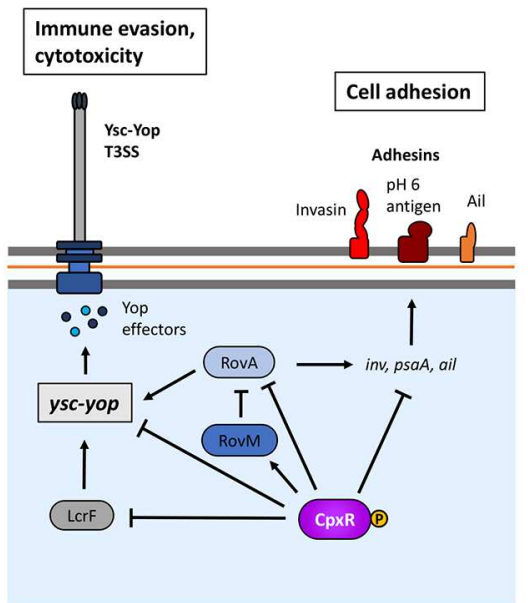

FIGURE 5 | The Cpx response is involved in the pathogenesis of several Gram-negative bacteria. The Cpx response regulates many different processes related to the pathogenesis of several different organisms, such as Escherichia coli (A), Salmonella enterica (B), Vibrio cholerae (C), and Yersinia spp. (D). Cpx regulation not only regulates the assembly of envelope-localized virulence determinants but also several other processes that impact envelope integrity and affect the ability of bacteria to colonize hosts. Proposed positive regulation is indicated by regular arrows, whereas blunt arrows represent negative regulation. Dashed lines indicate where regulation is indirect.

relation to the BFP of EPEC, despite their differing assembly and structural composition compared to P-pili (Nevesinjac and Raivio, 2005; Humphries et al., 2010; Vogt et al., 2010). In particular, efficient expression of the BFP was dependent on the Cpx-regulated periplasmic protein folding factors CpxP, DsbA, and DegP/HtrA (Vogt et al., 2010). The regulation of multiprotein envelope complexes by the Cpx response is not limited to pili, as flagella in several strains of $E$. coli are shown to be negatively regulated (De Wulf et al., 1999; Price and Raivio, 2009; Shimizu et al., 2016) and the Cpx response also regulates secretion machinery in several pathogenic E. coli (see below).

EPEC and EHEC are known to cause attaching and effacing $(\mathrm{A} / \mathrm{E})$ lesions on host intestinal tissue that are dependent on the locus of enterocyte effacement (LEE) T3SS (McDaniel et al.,
1995; McDaniel and Kaper, 1997). Activation of the Cpx response represses LEE T3S in EPEC (Macritchie et al., 2008). This regulation occurs through the repression of the transcription of several LEE operons and post-transcriptionally by the action of the periplasmic chaperone and protease DegP/HtrA (Macritchie et al., 2008; MacRitchie et al., 2012). The regulation of the LEE T3SS does have common features between EPEC and EHEC, namely the transcriptional regulator Ler (Mellies et al., 1999; Abe et al., 2008). However, recent studies in enterohaemorrhagic E. coli (EHEC) have led to contrasting conclusions as to the involvement of the Cpx response in regulating the LEE (De la Cruz et al., 2016; Shimizu et al., 2016). De la Cruz et al. (2016) found that increased levels of phosphorylated CpxR in the absence of CpxA repressed levels of LEE5-encoded EspABD 
translocator proteins as well as transcription of ler, in a manner dependent on the protease Lon. Overexpressing NlpE lead to a reduction in espA transcript as quantified by qPCR. However, Shimizu et al. (2016) found that espA-luxE reporter activity was activated in response to adhesion to hydrophobic glass beads and undifferentiated Caco- 2 cells in a manner dependent on NlpE and CpxA. In adhered cells, NlpE activated espA transcription (Shimizu et al., 2016). This led to the suggestion that activating the Cpx response after surface sensing by NlpE leads to an upregulation of LEE T3SS genes, specifically by CpxR regulation of the positive regulator LhrA (Shimizu et al., 2016). It is possible that the difference between these two studies is due to the fact that in most experiments, surface-adhered EHEC cells were used in Shizimu and colleagues' study, whereas the study of De la Cruz et al. was conducted mostly on cells grown in liquid media. More work should be conducted to elucidate the impact that surface sensing has on modulating Cpx signaling, as it appears that adhesion significantly alters the Cpx regulation of virulence in EHEC.

A growing body of evidence highlights the relevance of the Cpx response to pathogenesis beyond protein quality control in the envelope. Studies of the Cpx regulon in E. coli have shown that several genes involved in cell wall modifications are upregulated by Cpx activation (Raivio et al., 2013; Bernal-Cabas et al., 2015). These include D-alanyl-D-alanine carboxypeptidase DacC (penicillin binding protein (PBP) 6) (Pedersen et al., 1998), L,D-transpeptidase LdtD (Magnet et al., 2008), lytic transglycosylase Slt (Höltje et al., 1975) and YgaU, a hypothetical protein with a LysM domain predicted to be involved in cell wall degradation (Buist et al., 2008). Accordingly, the Cpx response is activated when $y g a U$ and/or $l d t D$ are deleted and in strains that lack PBPs 4, 57 and AmpH (Evans et al., 2013; Bernal-Cabas et al., 2015). Activating the Cpx response increased diaminopimelic acid (DAP)-DAP crosslinks formed by LdtD, suggesting that Cpx activation has direct ramifications on the structural composition of PG (Bernal-Cabas et al., 2015).

It was recently shown that deleting $c p x R$ increased susceptibility to the $\beta$-lactam antibiotic mecillinam, but constitutive activation led to defects in cell shape, growth, and division (Delhaye et al., 2016). Moreover, these defects were dependent on the expression of LdtD (Delhaye et al., 2016). These results point to a role for the Cpx response in monitoring not only protein quality control in the envelope, but also as a key regulatory factor for proper cell wall integrity. It has been observed that the Cpx response regulates amidases in $P$. aeruginosa and $S$. Typhimurium, suggesting that Cpx regulation of cell wall homeostasis may be a conserved function across Gram-negative organisms (Weatherspoon-Griffin et al., 2011; Yakhnina et al., 2015). The cell wall is essential for maintaining cell shape and preventing lysis during shifting osmotic conditions and is an important target for many antibiotics and innate immune strategies. Upregulation of PG-modifying enzymes may serve to reinforce the envelope during stress in other compartments of the envelope so as to increase its overall stability. Interestingly, it was recently shown that increased levels of DAP-DAP crosslinkages increases resistance to lysozyme (Stankeviciute et al., 2019). It is possible that Cpx-regulation of $l d t D$ and $y g a U$ may be a strategy for dealing with envelope stress arising from host innate immunity. Although the full picture remains to be seen, these studies point to the Cpx response as a fine-tuning regulator of PG structure that serves to mitigate potential stresses to the cell wall, both in and out of hosts.

\section{Citrobacter rodentium}

The presence of shared virulence determinants, most notably the LEE, in the murine pathogen Citrobacter rodentium, has led to its use as a model to study EPEC and EHEC infections in vivo (reviewed in Collins et al., 2014). Accordingly, the in vivo contribution of the Cpx response to virulence is arguably best demonstrated by recent studies in $C$. rodentium. The role of the Cpx response in promoting in vivo fitness and virulence has also previously been demonstrated in other organisms, such as in uropathogenic E. coli (Debnath et al., 2013), Salmonella (Fujimoto et al., 2018), and gonococcal Neisseria, where MisRS, a 2CST system suggested to be the Neisseria CpxRA homolog, was needed to establish infection in the genital tract of mice (Kandler et al., 2016; Gangaiah et al., 2017).

Deleting $\operatorname{cpxRA}$ attenuates the ability of $C$. rodentium to colonize and cause death in mice, in some cases completely (Thomassin et al., 2015, 2017; Vogt et al., 2019). cpx gene expression is induced during infection suggesting that the response is activated during host colonization (Thomassin et al., 2015 , 2017). Deleting $c p x P$ or $n l p E$ had no significant impact on colonization, virulence or ability to bind to HeLa cells unlike the cpxRA null mutant which was attenuated in all these aspects (Giannakopoulou et al., 2018). Attenuation of virulence was found to be independent of type III secretion and growth rate, as secretion profiles and growth rates remain relatively the same as compared to wildtype in $c p x R A$ mutants (Thomassin et al., 2015; Vogt et al., 2019).

Interestingly, it was recently reported that deleting the Cpxregulated genes $\operatorname{deg} P / h t r A$ and $d s b A$ led to a reduction in secreted protein levels of the T3SS translocator protein EspB in C. rodentium (Vogt et al., 2019). Furthermore, deleting degP or $d s b A$ caused $C$. rodentium to become completely avirulent, unlike $c p x R A$, which in this study resulted in less attenuation of virulence compared to previous studies (Thomassin et al., 2015, 2017; Vogt et al., 2019). The reason for the difference between these studies is currently unknown. However, mutating the promoter of $\operatorname{deg} P$ and $d s b A$ such that they are no longer under the control of CpxR does not attenuate virulence, suggesting that Cpx regulation of $\operatorname{deg} P$ or $d s b A$ is not the entire reason why these mutants are avirulent (Vogt et al., 2019). Overall, these studies point to a key role for the Cpx response in promoting in vivo fitness and virulence, but further work characterizing the mechanism of how this occurs is needed.

\section{Salmonella}

The Cpx response has been strongly implicated in the regulation of virulence in Salmonella enterica, which include S. enterica serovar Typhi and Typhimurium ( $S$. Typhi and $S$. Typhimurium, respectively; Figure 5B). In both $S$. Typhi and Typhimurium, 
virulence is heavily dependent on two pathogenicity islands SPI1 and SPI-2, both of which encode T3SS that facilitate invasion, immune invasion and intracellular survival (reviewed in McGhie et al., 2009; Que et al., 2013; Jennings et al., 2017). cpx genes in Salmonella are organized as they are in E. coli (Nakayama et al., 2003). The sequences of $c p x R A$ and $c p x P$ have 97,96 , and $88 \%$ identity, respectively, to their corresponding homologs in $E$. coli (Nakayama et al., 2003). As in E. coli, overexpression of the OM lipoprotein NlpE activates the Cpx response (Humphreys et al., 2004). CacA (Cpx-activating connector-like factor A), a small protein conserved in several Gram-negative species including E. coli, is induced by RpoS and is needed for full expression of Cpx-dependent genes such as cpxP and spy in Salmonella, suggesting that fine-tuning of $\mathrm{Cpx}$ regulation occurs by input from other regulatory systems, for example, in response to growth phase (Kato et al., 2012). Similarly, in E. coli, RpoS positively regulates $c p x R A$, further suggesting that $\mathrm{Cpx}$ regulation occurs in conjunction with other regulatory systems (De Wulf et al., 1999).

Transposon insertions in $c p x A$ were identified in a screen of mutants of $S$. Typhi with lowered ability to invade INT407 cells (LeClerc et al., 1998). In S. Typhimurium, deletion of $c p x A$ but not $c p x R$ at low $\mathrm{pH}(\mathrm{pH}$ 6.0) strongly represses the expression of a hilA-lacZ reporter, leading to a loss of invasioneffector protein expression and a severe defect in ability to invade INT407 cells. HilA is a key activator for SPI-1, which encodes T3SS responsible for secreting SipBCD translocator proteins and facilitating invasion (Que et al., 2013). These observations prompted the suggestion that CpxA repression of SPI-1 was CpxR-independent (Nakayama et al., 2003). However, several recent lines of evidence show that the regulation of SPI-1 by CpxA is not independent of CpxR. Deleting $c p x R A$ mimics the phenotype of $c p x R$ not $c p x A$ mutants and overexpressing NlpE represses the expression of SPI-1 (De la Cruz et al., 2015; Subramaniam et al., 2019). Further, deleting the AckA-Pta (acetate kinase, phosphotransacetylase) pathway responsible for generating acetyl phosphate mitigates the effect of deleting $c p x A$, suggesting that in the absence of CpxA phosphatase activity, non-specific phosphodonors activate CpxR, a phenomenon previously noted (McCleary and Stock, 1994; Danese and Silhavy, 1998; Wolfe et al., 2008; De la Cruz et al., 2015). The repression of SPI-1 by phosphorylated CpxR was dependent on Lon, a protease that degrades HilD, a positive regulator of HilA, suggesting that Cpx regulation of SPI-1 occurs by regulating the stability of HilA (De la Cruz et al., 2015). Recently, a study of the Cpx regulon in $S$. Typhimurium suggested that $p o c R$, a negative regulator of the $p d u$-cob cluster that encodes for genes involved in the breakdown of 1,2-propanediol (PDO), is CpxR-regulated (Subramaniam et al., 2019). PDO has been shown to repress hilA (Nakayama and Watanabe, 2006), suggesting that Cpx regulation of SPI-1 may occur with inputs from metabolism, although the precise mechanism of how this occurs is not clear.

Activation of the Cpx response was also shown to repress SsrB, a key activator for SPI-2 found on SPI-2 itself (Yoon et al., 2009; De la Cruz et al., 2015). It appears that this repression of SPI-2 may occur through two mechanisms, by CpxR directly binding the promoter of $s s r B$ (Subramaniam et al., 2019) and by the repression of HilD, which has been shown to activate both SPI-1 and SPI-2 (Bustamante et al., 2008; De la Cruz et al., 2015). Interestingly, CpxR positively regulates motility in Salmonella, a finding that stands in contrast to observations in $E$. coli (Subramaniam et al., 2019).

Antimicrobial peptides (AMPs) are among the many challenges enteric pathogens face as they transition to the intestinal environment. These AMPs cause stress primarily by disrupting the envelope, and in particular, disrupt important processes such as ATP generation (reviewed in Li et al., 2017). It makes sense, then, that the Cpx response is important for mediating resistance to various antimicrobial compounds. The Cpx response is activated by polymyxin B (Fujimoto et al., 2018). CpxR was found to regulate $p g t E$, an OM protease implicated in cleaving and inactivating antimicrobial peptides and deleting $c p x R$ accordingly increased sensitivity to polymyxin B (Subramaniam et al., 2019). NlpE overexpression increased resistance to antimicrobial peptides protamine, magainin 2 and melittin by upregulating two twin-arginine transport (Tat)dependent $\mathrm{N}$-acetyl muramoyl-L-alanine amidases AmiA and AmiC in a CpxR-dependent manner (Weatherspoon-Griffin et al., 2011). As these AMPs are an important component of innate immunity, overcoming this barrier is key for pathogens attempting to establish an infection. It appears that the Cpx response may play an important role in overcoming this challenge, a suggestion supported by other studies that have implicated the Cpx response in mediating resistance to AMPs, such as studies of MisRS, a CpxRA homolog in Neisseria spp. (Kandler et al., 2016).

It was initially reported that deleting $c p x R$ did not affect colonization of mice organs relative to WT (Humphreys et al., 2004). Interestingly, a recent in vivo study in a streptomycintreated mouse model found that while CpxRA was not needed to cause colitis, it was needed for colonization (Fujimoto et al., 2018). It is likely that the differences between these studies are due to the mouse model used in their experiments. While streptomycin-treated mice infected with $S$. Typhimurium cause symptoms analogous to the gastroenteritis caused by $S$. Typhimurium in humans, non-cleared mice infected with $S$. Typhimurium display a typhoid-like illness (Barthel et al., 2003). Further in vivo work characterizing the contribution of the Cpx response to Salmonella virulence is needed to elucidate the causes of the differences between these models of infection.

\section{Vibrio cholerae}

Vibrio cholerae is a Gram-negative enteric pathogen and the causative agent of the diarrheal disease cholera (reviewed in Clemens et al., 2017). In recent years, a number of studies have examined the impact of the Cpx response in $V$. cholerae. The basic genomic organization of $c p x$ genes resembles that of $E$. coli and S. enterica: $c p x R A$ is encoded as an operon with $c p x P$ encoded upstream and transcribed divergently (Slamti and Waldor, 2009). The sequences of $c p x A, c p x R$ and $c p x P$ possess $43.6,60.3$, and $21.6 \%$ identity, respectively, to their E. coli counterparts (Slamti and Waldor, 2009). Importantly, the degree of conservation in the predicted periplasmic sensing domain of $c p x A$ is far lower (20.7\%) compared to the cytoplasmic region responsible for 
CpxR phosphorylation (54.3\%), suggesting potential differences in CpxA-activating signals (Slamti and Waldor, 2009). Unlike in E. coli, NlpE in $V$. cholerae is type II (lacking a C-terminal domain) (Hirano et al., 2007) and NlpE overexpression does not activate the Cpx response in V. cholerae (Slamti and Waldor, 2009). Similarly, alkaline $\mathrm{pH}$ is also not a Cpx pathway inducer in $V$. cholerae (Acosta et al., 2015b). The Cpx response in $V$. cholerae is activated in response to $\mathrm{CuSO}_{4}$, chloride ions, aberrant disulfide bond formation, iron chelation, the absence of RND (resistance-nodulation-division) efflux pumps and ROS (Slamti and Waldor, 2009; Taylor et al., 2014; Acosta et al., 2015b; Kunkle et al., 2017). Mutations in DsbD, which is involved in the folding of secreted proteins by mediating disulfide bond formation (Ito and Inaba, 2008) and TolC, the OM component of efflux pumps (Koronakis et al., 2004), were enriched in a screen of mutants that activated the Cpx response, suggesting a conserved role for Cpx regulation of envelope homeostasis in $V$. cholerae (Slamti and Waldor, 2009).

The Cpx response has been implicated in regulating several processes that are important for the survival and growth of $V$. cholerae as it infects its host (Figure 5C). An important innate immune strategy is the sequestration of important minerals to limit bacterial growth. Iron, in particular, is key to several metabolic processes and is normally limited in hosts as it is sequestered in heme groups or iron-carrying proteins such as ferritin. As such, iron uptake presents an important challenge for enteric pathogens to overcome to establish infection (reviewed in Hood and Skaar, 2012). The Cpx response has been implicated in adapting to the stresses caused by low iron. The Cpx response in $V$. cholerae is activated in response to the chelation of iron and the Cpx regulon in V. cholerae O1 El Tor C6706 is enriched in genes involved in iron acquisition and metabolism, such as those involved in biosynthesis of the siderophore vibriobactin, ferrichrome transport and heme uptake (Acosta et al., 2015b). Furthermore, supplementing growth media with an excess of $\mathrm{FeSO}_{4}$ was able to decrease activation of the response not only in iron-limiting conditions by 2,2'-bipyridyl, but also in response to diamide and RND efflux pump deletions suggesting that these Cpx-activating cues are related in some way to iron uptake (Acosta et al., 2015b).

$V$. cholerae possess six RND efflux systems, VexAB, CD, $\mathrm{EF}, \mathrm{GH}, \mathrm{IJK}$, and LM, all of which likely utilize the OM protein TolC as their OM pore (Bina et al., 2008). These efflux pumps are responsible for the efflux of a variety of potentially harmful substances, including antibiotics such as polymyxin B, erythromycin and ampicillin and detergents such Triton X-100 and sodium dodecyl sulfate (SDS) (Bina et al., 2008; Taylor et al., 2012). Loss of these RND efflux pumps leads to a reduction in cholera toxin (CT) and toxin coregulated pilus (TCP) production and abolishes $V$. cholerae's ability to colonize in an infant mouse model (Bina et al., 2008). Deletions of tolC and genes encoding RND efflux pumps VexAB and VexGH activate the Cpx response and likewise, activating the $\mathrm{Cpx}$ response induces expression of TolC and VexAB and VexGH (Slamti and Waldor, 2009; Taylor et al., 2014; Acosta et al., 2015b). These results strongly link the Cpx response to V. cholerae's efflux machinery. However, although inducing the $\mathrm{Cpx}$ response by $\mathrm{KCl}$ or by a $c p x A^{*}$ mutation was able to increase growth on thiosulfate-citrate-bile sucrose (TCBS) agar, a medium which requires the action of RND efflux to allow for growth, deleting $c p x R$ did not negatively affect growth on TCBS agar relative to WT suggesting that the Cpx response is not normally required for growth in efflux-requiring conditions (Taylor et al., 2014). Thus, although efflux and the Cpx response are strongly linked genetically, the extent to which the Cpx response is linked to innate antimicrobial resistance in $V$. cholerae remains to be determined.

Interestingly, the significance of Cpx regulation of RND efflux pumps was shown to be connected to iron acquisition and transport (Kunkle et al., 2017). Mutations in genes involved in the synthesis of vibriobactin, a catechol siderophore (Griffiths et al., 1984), were found to suppress Cpx activation in efflux-deficient mutants (Kunkle et al., 2017). Levels of extracellular vibriobactin were reduced in RND efflux negative mutants, suggesting RND efflux pumps function to transport vibriobactin out of the cell and that in their absence, vibriobactin aberrantly accumulates in the periplasmic space (Kunkle et al., 2017). As iron is an essential component of several IM complexes of the electron transport chain (ETC) (Friedrich et al., 2016), it was suggested that the activation of the Cpx response in the RND-deficient mutant was due to the chelation of iron away from these complexes, leading to aberrant protein folding at the IM and/or the production of ROS during respiration (Kunkle et al., 2017). Consonant with this hypothesis is the observation that activation in efflux-negative mutants was abolished in anaerobic growth and in a $s d h A$ mutant, which encodes for a subunit of succinate dehydrogenase (Kunkle et al., 2017). Studies in EPEC have shown that respiratory complexes found at the IM, such as NDH-I and cytochrome $b o_{3}$ are negatively regulated by the $\mathrm{Cpx}$ response, suggesting that monitoring respiratory complexes at the IM may be a conserved function of the Cpx response, although it remains to be seen how the Cpx response senses these stresses (Raivio et al., 2013; Guest et al., 2017).

Elucidating the precise role of the Cpx response in regulating virulence in $V$. cholerae is complicated by contrasting results in different, albeit closely related strains. The primary diarrheagenic effect of $V$. cholerae stems from the ADP-ribosylating action of $\mathrm{CT}$, which leads to changes in intracellular signaling and fluid secretion from intestinal epithelial cells (Field et al., 1972; and Gill et al., 1978). RND efflux pumps have been shown to be needed for the optimal production of CT and TCP in O1 El Tor strain N16961, but deleting $c p x R$ in a strain lacking all six RND efflux pumps did not affect decreased levels of CT and TCP, suggesting a Cpx-independent mechanism for the regulation of CT and TCP production (Bina et al., 2008; Taylor et al., 2014). Furthermore, neither $c p x A^{*}$ (constitutively active) or $c p x R$ mutants changed CT or TCP levels relative to WT (Taylor et al., 2014). Accordingly, neither deletions of $c p x R, c p x A$ or $c p x P$ or activation of the response via a $c p x A^{*}$ allele affected the ability of N16961 to colonize infant mice (Slamti and Waldor, 2009).

In contrast to these results, mutating $c p x R$ in a related O1 El Tor strain, C6706, led to an increase in $\operatorname{ctxB}$, tcpA, and $t c p P$ transcription (Acosta et al., 2015a). Correspondingly, overexpressing CpxR led to a complete abrogation of the expression of CT and TCP both at the transcriptional and protein 
level (Acosta et al., 2015a). CpxR overexpression repressed the transcription of ToxT, the direct regulator of TCP and CT (DiRita et al., 1991) and TcpP, a regulator involved in promoting the expression of ToxT (Häse and Mekalanos, 1998), suggesting that Cpx regulation of virulence occurs through established virulence regulators (Acosta et al., 2015a). It was suggested that the discrepancy in results between C6706 and N16961 could be due to a known defect in the ability of N16961 to quorum sense, another process closely involved in regulating virulence in $V$. cholerae (Zhu et al., 2002). Nonetheless, these contrasting results underscore the need to be cognizant of strain differences when conducting work on even closely related strains and future studies investigating these differences may help elucidate the precise mechanisms of Cpx regulation of key $V$. cholerae virulence determinants.

\section{Yersinia spp.}

The genus Yersinia include a number of Gram-negative pathogens including $Y$. pestis, $Y$. pseudotuberculosis and $Y$. enterolitica. Studies of the Cpx response have focused on the latter two species, Y. pseudotuberculosis and Y. enterolitica, which, while not as well-known as the infamous cause of the bubonic and pneumonic plagues, are nonetheless significant pathogens of humans (Drummond et al., 2012). However, a study in $Y$. pestis has implicated the Cpx response in promoting survival in neutrophils (O'Loughlin et al., 2010), and as such it is certainly possible that the findings in other species of Yersinia are applicable to $Y$. pestis.

The virulence of Yersinia spp. depends heavily on the action of the virulence plasmid-encoded Ysc-Yop (Yersinia secretionYersinia outer protein) T3SS. Ycs proteins form the machinery responsible for the translocation of a collection of different Yop effectors that block phagocytosis into host cells directly and allow for extracellular replication of Yersinia (reviewed in Cornelis, 2002). Yersinia spp. are thought to primarily be extracellular pathogens, although they possess the ability to invade and survive intracellularly (Grabenstein et al., 2004). Several ESRs contribute to virulence factor regulation in Yersinia, including the Psp and Rcs responses (Flores-Kim and Darwin, 2012; Li et al., 2015b).

In the last two decades, several studies have examined the Cpx response in $Y$. enterolitica and $Y$. pseudotuberculosis, particularly in relation to type III secretion and adhesion to host cells (Figure 5D). The sequence identity of $c p x R$ and $c p x A$ to their E. coli homologs are high in both $Y$. pseudotuberculosis and $Y$. enterolitica (89 and 80\%, respectively in Y. pseudotuberculosis and 81 and 74\%, respectively in Y. enterolitica) (Heusipp et al., 2004; Carlsson et al., 2007a). As in E. coli, the Cpx regulon in Yersinia spp. includes envelope protein folding factors and proteases such as $h t r A / \operatorname{deg} P, d s b A$ and ppiA (Heusipp et al., 2004; Carlsson et al., 2007a,b; Liu et al., 2011).

Several studies have shown that deleting $c p x A$ represses the production of the Ysc secretion machinery, Yop effectors as well as adhesins such as invasin (inv) in Y. pseudotuberculosis (Carlsson et al., 2007a,b; Liu et al., 2011, 2012). Furthermore, deleting cpxA abrogates the ability of $Y$. pseudotuberculosis to cause cytotoxicity in HeLa cells caused by YopE, a type III secretion secreted cytotoxin (Carlsson et al., 2007a,b; Vlahou et al., 2009; Liu et al., 2012). This repression of virulence occurs in the $c p x A$ mutant as a result of non-specific phosphorylation of CpxR in the absence of CpxA phosphatase activity, as constitutively active $c p x A^{*}$ mutants produce similar results to $c p x A$ knock-out mutants, and removing acetyl-phosphate by introducing a $\triangle a c k A$, pta mutation abolishes the effects of deleting cpxA (Carlsson et al., 2007a,b; Liu et al., 2011, 2012). Cytotoxicity was not affected in a $c p x R$ null mutant and this mutation actually increased cell adhesion and invasion (Carlsson et al., 2007a,b). Furthermore, ectopically expressing CpxR, but not CpxR D51A (unable to be phosphorylated), repressed cytotoxicity, indicating that phosphorylated CpxR is responsible for the repression of cell adhesion and type III secretion (Carlsson et al., 2007a; Liu et al., 2011). These results suggest that the Cpx response functions to repress T3SS assembly and secretion and are corroborated by transcriptional data which shows that $c p x R$ is downregulated during conditions that induce the expression of the Ysc-Yops T3SS (Carlsson et al., 2007a).

The precise mechanism of regulation of these processes appears to require a multitude of different regulatory approaches. Although Ysc structural protein levels are reduced in membrane fractions in $c p x A$ null mutants, the promoters of these genes lack CpxR binding sites, suggesting indirect regulation at the transcriptional level or potential post-transcriptional regulation by other Cpx-induced factors (Carlsson et al., 2007a; Liu et al., 2012). In contrast, the transcription of several yop effector genes and syc effector chaperones are directly regulated by phosphorylated CpxR (Liu et al., 2012). In addition, LcrF, a transcription factor which induces the expression of yop genes when the temperature shifts from 26 to $37^{\circ} \mathrm{C}$ (Cornelis et al., 1989), is also directly regulated by CpxR suggesting that regulation of Yop effectors occurs not only through directly modifying transcription but also by modulating expression of other transcriptional activators (Liu et al., 2012).

A similar story can be told in regards to cell adhesion. Phosphorylated CpxR can directly modulate transcription of known adhesins invasin and $\mathrm{pH} 6$ antigen ( $p s a A)$, as well as proposed adhesins such as the OM protein Ail, suggesting direct regulation (Carlsson et al., 2007b; Liu et al., 2011). However, regulation of these factors also occurs through other regulators. RovA, a global transcriptional regulator that activates the expression of invasin, is directly regulated by CpxR (Carlsson et al., 2007b). A further level of control was recently discovered when it was demonstrated that RovM, a regulator of RovA that represses its expression during nutrient limitation, was found to be directly CpxR-P regulated (Heroven and Dersch, 2006; Heroven et al., 2008; Thanikkal et al., 2019). Thus, RovA expression is not only modulated by CpxR at its own promoter, but also through CpxR upregulation of RovM. The in vivo significance of this result may be related to the finding that the RovM regulatory cascade is implicated in causing a lifestyle switch from acute to persistent infection in mice (Avican et al., 2015). This is also consistent with findings in S. Typhimurium, where the Cpx response was dispensable for causing acute virulence in mice, but was needed to colonize the mouse gut over longer spans of time (Fujimoto et al., 2018). The sum of these results points to a network of regulatory connections that control 
the expression of several key Yersinia virulence determinants. An area for further investigation is elucidating the precise inducing cues of the Cpx response in Yersinia spp. so as to understand why the Cpx response negatively regulates key envelope localized virulence determinants.

The Cpx response in $Y$. enterolitica has not been explored as thoroughly as in its sister species. Nonetheless, studies point to some key differences. Unlike in Y. pseudotuberculosis, to our knowledge, no studies have been published successfully deleting cpxA in $Y$. enterolitica, and attempts to do so even in the presence of CpxA expression in trans have not been successful (Ronnebaumer et al., 2009). Furthermore, overexpression of CpxA is deleterious even in normal growth conditions, suggesting that the effect of deleting and overexpressing CpxA is related to the phosphorylation of CpxR (Ronnebaumer et al., 2009). In Y. pseudotuberculosis, however, deleting cpxA only causes a minor growth defect (Carlsson et al., 2007a). At this point, it is unclear what is responsible for the apparent differences between $Y$. pseudotuberculosis and Y. enterolitica. However, as the Cpx regulon remains relatively uncharacterized in both of these species, its further characterization may yield insights into the root of these differences.

\section{Summary}

It should be noted that studies of the Cpx response are not limited to the organisms we discuss in this review. Unfortunately, given restraints on space, it is impossible to discuss all of the relevant work in other organisms. As such, we wish to point readers to the following studies for further reading: Shigella spp. (Nakayama and Watanabe, 1995, 1998; Mitobe et al., 2005), Legionella pneumophila (Gal-Mor and Segal, 2003; Vincent et al., 2006; Altman and Segal, 2008), Haemophilus ducreyi (Labandeira-Rey et al., 2009, 2010; Gangaiah et al., 2013), and Neisseria spp. (Tzeng et al., 2004, 2008; Kandler et al., 2016; Gangaiah et al., 2017).

The work conducted in a diverse array of different organisms highlights the contribution of the Cpx response to the virulence of several Gram-negative pathogens. Although the exact details can differ between organisms, or even closely related strains, there are a few important motifs that we see repeated throughout these studies. The Cpx regulon across species typically includes envelope-localized protein folding and degradation factors, such as DegP/HtrA. There exists a consistent link between envelope-localized, multi-protein complexes, such as pili or secretion machinery, and regulation by the Cpx response. These patterns support an overarching paradigm where the Cpx response functions to maintain envelope integrity by monitoring protein quality control. However, the specific effect of the Cpx response in different organisms is not uniform, despite the fact that essentially all of the organisms surveyed in this review are enteric pathogens that, generally speaking, share a similar niche in the host. In some organisms (such as $C$. rodentium), the Cpx response is indispensable to colonization and virulence in vivo, while in other organisms, activation of the Cpx response has a predominantly negative impact on key virulence determinants. Related to this, while in general, the Cpx response negatively regulates key virulence determinants, the presence of the Cpx response tends to be positively associated with colonization or long-term persistence. Given this general observation, it appears that the role of the Cpx response in pathogenesis tends not toward promoting virulence factor expression, but rather ensuring that the cell can survive in the midst of the multitude of stresses faced during the course of infection, whether that be due to the expression of the virulence factors themselves or from external factors, such as host immune strategies.

What is responsible for the observed differences between organisms? It is almost certain that phylogenetic differences can explain aspects of the observed diversity. For example, remarkable similarities exist in the mechanism of Cpx regulation of the LEE T3SS in pathogenic E. coli and the SPI-1 T3SS in $S$. Typhimurium (see Figures 4, 5 and the corresponding sections). But when comparing more distantly related species (as is reflected in greater sequence dissimilarity in $c p x$ genes), such as $E$. coli and $V$. cholerae, more disparities tend to be seen in inducing cues, mechanisms of signaling or regulon members. It is also highly likely that differences in life-cycle and infection strategies influence how the Cpx response functions in different species. For example, while phylogenetically, E. coli may be more closely related to Salmonella, than to $V$. cholerae, the extracellular lifestyle adopted by EPEC and EHEC is more analogous to $V$. cholerae than to the invasive lifestyle of Salmonella. And so, commonalities, such as the regulation of pilus expression, can be seen in E. coli and V. cholerae. Perhaps part of the issue is that the Cpx response is best characterized in various strains of $E$. coli and studies in other pathogens have yet to be conducted to the same level of depth. If this is the case, further study in these organisms will only yield expanded insight into our understanding of the Cpx response and its function in the physiology and pathogenesis of a variety of different organisms.

\section{CONCLUDING REMARKS}

It is clear from the literature that ESRs such as the $\sigma^{\mathrm{E}}$ response and the Cpx response play key roles in the physiology and pathogenesis of several Gram-negative bacteria. Nonetheless, it is also clear that many questions remain about these ESRs, such as those about precise mechanisms of signaling and regulation of target genes and processes, and their relevance to in vivo fitness. Particularly of interest is exploring the precise points at which these ESRs are relevant to colonization and infection. When and where are these ESRs active? What precise cues in vivo induce these responses? Do ESRs predominantly mitigate encountered stresses or do ESRs play more proactive roles in promoting colonization or virulence? Exploring these questions further will not only increase our basic understanding of these Gram-negative bacteria but also may help provide potential strategies for the prevention and treatment of the diseases caused by these organisms.

\section{AUTHOR CONTRIBUTIONS}

$\mathrm{CH}$ and TC reviewed the literature, co-wrote the manuscript, and prepared the figures. GR and TR conceived the idea and co-edited the drafts. 


\section{FUNDING}

This work was funded by operating grant MOP 342982 from the Canadian Institutes of Health Research and discovery grant

\section{REFERENCES}

Abe, H., Miyahara, A., Oshima, T., Tashiro, K., Ogura, Y., Kuhara, S., et al. (2008). Global regulation by horizontally transferred regulators establishes the pathogenicity of Escherichia coli. DNA Res. 15, 25-38. doi: 10.1093/dnares/dsm033

Acosta, N., Pukatzki, S., and Raivio, T. L. (2015a). The Cpx system regulates virulence gene expression in Vibrio cholerae. Infect. Immun. 83, 2396-2408. doi: 10.1128/IAI.03056-14

Acosta, N., Pukatzki, S., and Raivio, T. L. (2015b). The Vibrio cholerae Cpx envelope stress response senses and mediates adaptation to low iron. $J$. Bacteriol. 197, 262-276. doi: 10.1128/JB.01957-14

Ades, S. E., Connolly, L. E., Alba, B. M., and Gross, C. A. (1999). The Escherichia coli sigma(E)-dependent extracytoplasmic stress response is controlled by the regulated proteolysis of an anti-sigma factor. Genes Dev. 13, 2449-2461. doi: 10.1101/gad.13.18.2449

Ahuja, N., Korkin, D., Chaba, R., Cezairliyan, B. O., Sauer, R. T., Kim, K. K., et al. (2009). Analyzing the interaction of RseA and RseB, the two negative regulators of the sigmaE envelope stress response, using a combined bioinformatic and experimental strategy. J. Biol. Chem. 284, 5403-5413. doi: $10.1074 / j b c . M 806012200$

Akiyama, Y. (2009). Quality control of cytoplasmic membrane proteins in Escherichia coli. J. Biochem. 146, 449-454. doi: 10.1093/jb/mvp071

Alba, B. M., Leeds, J. A., Onufryk, C., Lu, C. Z., and Gross, C. A. (2002). DegS and YaeL participate sequentially in the cleavage of RseA to activate the sigma(E)-dependent extracytoplasmic stress response. Genes Dev. 16, 2156-2168. doi: 10.1101/gad.1008902

Altman, E., and Segal, G. (2008). The response regulator CpxR directly regulates expression of several Legionella pneumophila icm/dot components as well as new translocated substrates. J. Bacteriol. 190, 1985-1996. doi: 10.1128/JB.01493-07

Amar, A., Pezzoni, M., Pizarro, R. A., and Costa, C. S. (2018). New envelope stress factors involved in sigma(E) activation and conditional lethality of rpoE mutations in Salmonella enterica. Microbiology 164, 1293-1307. doi: 10.1099/mic.0.000701

Amy, M., Velge, P., Senocq, D., Bottreau, E., Mompart, F., and Virlogeux-Payant, I. (2004). Identification of a new Salmonella enterica serovar Enteritidis locus involved in cell invasion and in the colonisation of chicks. Res. Microbiol. 155, 543-552. doi: 10.1016/j.resmic.2004.03.005

Appia-Ayme, C., Hall, A., Patrick, E., Rajadurai, S., Clarke, Thomas, A., and Rowley, G. (2012). ZraP is a periplasmic molecular chaperone and a repressor of the zinc-responsive two-component regulator ZraSR. Biochem. J. 442, 85-93. doi: 10.1042/BJ20111639

Appia-Ayme, C., Patrick, E., Sullivan, M. J., Alston, M. J., Field, S. J., AbuOun, M., et al. (2011). Novel inducers of the envelope stress response BaeSR in Salmonella Typhimurium: BaeR is critically required for tungstate waste disposal. PLOS ONE 6:e23713. doi: 10.1371/journal.pone. 0023713

Arie, J. P., Sassoon, N., and Betton, J. M. (2001). Chaperone function of FkpA, a heat shock prolyl isomerase, in the periplasm of Escherichia coli. Mol. Microbiol. 39, 199-210. doi: 10.1046/j.1365-2958.2001.02250.x

Asmar, A. T., and Collet, J.-F. (2018). Lpp, the Braun lipoprotein, turns 50major achievements and remaining issues. FEMS Microbiol. Lett. 365:fny199. doi: 10.1093/femsle/fny199

Audrain, B., Ferrieres, L., Zairi, A., Soubigou, G., Dobson, C., Coppee, J. Y., et al. (2013). Induction of the Cpx envelope stress pathway contributes to Escherichia coli tolerance to antimicrobial peptides. Appl. Environ. Microbiol. 79, 7770-7779. doi: 10.1128/AEM.02593-13

Avican, K., Fahlgren, A., Huss, M., Heroven, A. K., Beckstette, M., Dersch, P., et al. (2015). Reprogramming of Yersinia from virulent to persistent mode
RGPIN 238422-2013 from the Natural Sciences and Engineering Research Council to TR and a Norwich Research Park BBSRC Doctoral Training Partnership Studentship awarded to $\mathrm{CH}$ (BB/M011216/1).

revealed by complex in vivo RNA-seq analysis. PLoS Pathog. 11:e1004600. doi: 10.1371/journal.ppat.1004600

Baker, T. A., and Sauer, R. T. (2012). ClpXP, an ATP-powered unfolding and protein-degradation machine. Biochim. Biophys. Acta 1823, 15-28. doi: 10.1016/j.bbamcr.2011.06.007

Bardwell, J. C. A., McGovern, K., and Beckwith, J. (1991). Identification of a protein required for disulfide bond formation in vivo. Cell 67, 581-589. doi: 10.1016/0092-8674(91)90532-4

Barthel, M., Hapfelmeier, S., Quintanilla-Martinez, L., Kremer, M., Rohde, M., Hogardt, M., et al. (2003). Pretreatment of mice with streptomycin provides a Salmonella enterica serovar Typhimurium colitis model that allows analysis of both pathogen and host. Infect. Immun. 71, 2839-2858. doi: 10.1128/IAI.71.5.2839-2858.2003

Bazire, A., Shioya, K., Soum-Soutéra, E., Bouffartigues, E., Ryder, C., GuentasDombrowsky, L., et al. (2010). The sigma factor AlgU plays a key role in formation of robust biofilms by nonmucoid and Pseudomonas aeruginosa. J. Bacteriol. 192, 3001-3010. doi: 10.1128/JB.01633-09

Behrens, S., Maier, R., de Cock, H., Schmid, F. X., and Gross, C. A. (2001). The SurA periplasmic PPIase lacking its parvulin domains functions in vivo and has chaperone activity. EMBO J. 20, 285-294. doi: 10.1093/emboj/20.1.285

Belas, R. (2014). Biofilms, flagella, and mechanosensing of surfaces by bacteria. Trends Microbiol. 22, 517-527. doi: 10.1016/j.tim.2014.05.002

Bernal-Cabas, M., Ayala, J. A., and Raivio, T. L. (2015). The Cpx envelope stress response modifies peptidoglycan cross-linking via the L,Dtranspeptidase LdtD and the novel protein YgaU. J. Bacteriol. 197, 603-614. doi: 10.1128/JB.02449-14

Bina, X. R., Provenzano, D., Nguyen, N., and Bina, J. E. (2008). Vibrio cholerae RND family efflux systems are required for antimicrobial resistance, optimal virulence factor production, and colonization of the infant mouse small intestine. Infect. Immun. 76, 3595-3605. doi: 10.1128/IAI.01620-07

Bodelon, G., Marin, E., and Fernandez, L. A. (2009). Role of periplasmic chaperones and BamA (YaeT/Omp85) in folding and secretion of intimin from enteropathogenic Escherichia coli strains. J. Bacteriol. 191, 5169-5179. doi: 10.1128/JB.00458-09

Bos, M. P., Robert, V., and Tommassen, J. (2007). Biogenesis of the gramnegative bacterial outer membrane. Annu. Rev. Microbiol. 61, 191-214. doi: 10.1146/annurev.micro.61.080706.093245

Braun, V. (1975). Covalent lipoprotein from the outer membrane of Escherichia coli. Biochim. Biophys. Acta 415, 335-377. doi: 10.1016/0304-4157(75)90013-1

Buelow, D. R., and Raivio, T. L. (2005). Cpx signal transduction is influenced by a conserved N-terminal domain in the novel inhibitor CpxP and the periplasmic protease DegP. J. Bacteriol. 187, 6622-6630. doi: 10.1128/JB.187.19.6622-6630.2005

Buist, G., Steen, A., Kok, J., and Kuipers, O. P. (2008). LysM, a widely distributed protein motif for binding to (peptido)glycans. Mol. Microbiol. 68, 838-847. doi: 10.1111/j.1365-2958.2008.06211.x

Bustamante, V. H., Martínez, L. C., Santana, F. J., Knodler, L. A., SteeleMortimer, O., and Puente, J. L. (2008). HilD-mediated transcriptional crosstalk between SPI-1 and SPI-2. Proc. Natl. Acad. Sci. U. S. A. 105, 14591-14596. doi: 10.1073/pnas.0801205105

Button, J. E., Silhavy, T. J., and Ruiz, N. (2007). A suppressor of cell death caused by the loss of sigmaE downregulates extracytoplasmic stress responses and outer membrane vesicle production in Escherichia coli. J. Bacteriol. 189, 1523-1530. doi: 10.1128/JB.01534-06

Carlsson, K. E., Liu, J., Edqvist, P. J., and Francis, M. S. (2007a). Extracytoplasmicstress-responsive pathways modulate type III secretion in Yersinia pseudotuberculosis. Infect. Immun. 75, 3913-3924. doi: 10.1128/IAI.01346-06

Carlsson, K. E., Liu, J., Edqvist, P. J., and Francis, M. S. (2007b). Influence of the Cpx extracytoplasmic-stress-responsive pathway on Yersinia sp.-eukaryotic cell contact. Infect. Immun. 75, 4386-4399. doi: 10.1128/IAI.01450-06 
Chaba, R., Alba, B. M., Guo, M. S., Sohn, J., Ahuja, N., Sauer, R. T., et al. (2011). Signal integration by DegS and RseB governs the and\#963; E-mediated envelope stress response in Escherichia coli. Proc. Natl. Acad. Sci. U. S. A. 108, 2106-2111. doi: 10.1073/pnas.1019277108

Chaba, R., Grigorova, I. L., Flynn, J. M., Baker, T. A., and Gross, C. A. (2007). Design principles of the proteolytic cascade governing the sigmaE-mediated envelope stress response in Escherichia coli: keys to graded, buffered, and rapid signal transduction. Genes Dev. 21, 124-136. doi: 10.1101/gad.1496707

Chao, Y., and Vogel, J. (2016). A 3' UTR-derived small RNA provides the regulatory noncoding arm of the inner membrane stress response. Mol. Cell 61, 352-363. doi: 10.1016/j.molcel.2015.12.023

Chassaing, B., and Darfeuille-Michaud, A. (2013). The $\sigma \mathrm{E}$ pathway is involved in biofilm formation by Crohn's disease-associated adherent-invasive Escherichia coli. J. Bacteriol. 195, 76-84. doi: 10.1128/JB.01079-12

Clemens, J. D., Nair, G. B., Ahmed, T., Qadri, F., and Holmgren, J. (2017). Cholera. Lancet 390, 1539-1549. doi: 10.1016/S0140-6736(17)30559-7

Collinet, B., Yuzawa, H., Chen, T., Herrera, C., and Missiakas, D. (2000). RseB binding to the periplasmic domain of RseA modulates the RseA: $\varsigma$ E interaction in the cytoplasm and the availability of $\varsigma$ E.RNA polymerase. J. Biol. Chem. 275, 33898-33904. doi: 10.1074/jbc.M006214200

Collins, J. W., Keeney, K. M., Crepin, V. F., Rathinam, V. A., Fitzgerald, K. A., Finlay, B. B., et al. (2014). Citrobacter rodentium: infection, inflammation and the microbiota. Nat. Rev. Microbiol. 12, 612-623. doi: 10.1038/nrmicro3315

Coornaert, A., Lu, A., Mandin, P., Springer, M., Gottesman, S., and Guillier, M. (2010). MicA sRNA links the PhoP regulon to cell envelope stress. Mol. Microbiol. 76, 467-479. doi: 10.1111/j.1365-2958.2010.07115.x

Cornelis, G., Sluiters, C., Lambert de Rouvroit, C., and Michiels, T. (1989). Homology between VirF, the transcriptional activator of the Yersinia virulence regulon, and AraC, the Escherichia coli arabinose operon regulator. J. Bacteriol. 171, 254-262. doi: 10.1128/jb.171.1.254-262.1989

Cornelis, G. R. (2002). The Yersinia Ysc-Yop 'type III' weaponry. Nat. Rev. Mol. Cell Biol. 3, 742-752. doi: 10.1038/nrm932

Cosma, C. L., Danese, P. N., Carlson, J. H., Silhavy, T. J., and Snyder, W. B. (1995). Mutational activation of the Cpx signal transduction pathway of Escherichia coli suppresses the toxicity conferred by certain envelope-associated stresses. Mol. Microbiol. 18, 491-505. doi: 10.1111/j.1365-2958.1995.mmi_18030491.x

Crouch, M. L., Becker, L. A., Bang, I. S., Tanabe, H., Ouellette, A. J., and Fang, F. C. (2005). The alternative sigma factor sigma is required for resistance of Salmonella enterica serovar Typhimurium to anti-microbial peptides. Mol. Microbiol. 56, 789-799. doi: 10.1111/j.1365-2958.2005.04578.x

Dalebroux, Z. D., Matamouros, S., Whittington, D., Bishop, R. E., and Miller, S. I. (2014). PhoPQ regulates acidic glycerophospholipid content of the Salmonella Typhimurium outer membrane. Proc. Natl. Acad. Sci. U. S. A. 111, 1963-1968. doi: $10.1073 /$ pnas. 1316901111

Dalebroux, Z. D., and Miller, S. I. (2014). Salmonellae PhoPQ regulation of the outer membrane to resist innate immunity. Curr. Opin. Microbiol. 17, 106-113. doi: 10.1016/j.mib.2013.12.005

Damron, F. H., and Goldberg, J. B. (2012). Proteolytic regulation of alginate overproduction in Pseudomonas aeruginosa. Mol. Microbiol. 84, 595-607. doi: 10.1111/j.1365-2958.2012.08049.x

Danese, P. N., and Silhavy, T. J. (1998). CpxP, a stress-combative member of the Cpx regulon. J. Bacteriol. 180, 831-839.

Danese, P. N., Snyder, W. B., Cosma, C. L., Davis, L. J. B., and Silhavy, T. J. (1995). The Cpx two-component signal transduction pathway of Escherichia coli regulates transcription of the gene specifying the stress-inducible periplasmic protease, DegP. Genes Dev. 9, 387-398. doi: 10.1101/gad.9.4.387

Dartigalongue, C., Missiakas, D., and Raina, S. (2001). Characterization of the Escherichia coli sigma E regulon. J. Biol. Chem. 276, 20866-20875. doi: 10.1074/jbc.M100464200

Darwin, A. J. (2013). Stress relief during host infection: the phage shock protein response supports bacterial virulence in various ways. PLoS Pathog. 9:e1003388. doi: 10.1371/journal.ppat.1003388

De la Cruz, M. A., Morgan, J. K., Ares, M. A., Yanez-Santos, J. A., Riordan, J. T., and Giron, J. A. (2016). The two-component system CpxRA negatively regulates the locus of enterocyte effacement of enterohemorrhagic Escherichia coli involving sigma and lon protease. Front. Cell. Infect. Microbiol. 6:11. doi: 10.3389/fcimb.2016.00011
De la Cruz, M. A., Perez-Morales, D., Palacios, I. J., Fernandez-Mora, M., Calva, E., and Bustamante, V. H. (2015). The two-component system CpxR/A represses the expression of Salmonella virulence genes by affecting the stability of the transcriptional regulator HilD. Front. Microbiol. 6:807. doi: 10.3389/fmicb.2015.00807

De Las Peñas, A., Connolly, L., and Gross, C. A. (1997a). SigmaE is an essential sigma factor in Escherichia coli. J. Bacteriol. 179, 6862-6864. doi: $10.1128 / \mathrm{jb} .179 .21 .6862-6864.1997$

De Las Peñas, A., Connolly, L., and Gross, C. A. (1997b). The sigmaE-mediated response to extracytoplasmic stress in Escherichia coli is transduced by RseA and RseB, two negative regulators of sigmaE. Mol. Microbiol. 24, 373-385. doi: 10.1046/j.1365-2958.1997.3611718.x

De Wulf, P., Kwon, O., and Lin, E. C. C. (1999). The CpxRA signal transduction system of Escherichia coli: growth-related autoactivation and control of unanticipated target operons. J. Bacteriol. 181, 6772-6778.

Debnath, I., Norton, J. P., Barber, A. E., Ott, E. M., Dhakal, B. K., Kulesus, R. R., et al. (2013). The Cpx stress response system potentiates the fitness and virulence of uropathogenic Escherichia coli. Infect. Immun. 81, 1450-1459. doi: 10.1128/IAI.01213-12

Delhaye, A., Collet, J. F., and Laloux, G. (2016). Fine-tuning of the Cpx envelope stress response is required for cell wall homeostasis in Escherichia coli. MBio 7:e00047-16. doi: 10.1128/mBio.00047-16

Delhaye, A., Laloux, G., and Collet, J. F. (2019). The lipoprotein NlpE is a Cpx sensor that serves as a sentinel for protein sorting and folding defects in the Escherichia coli envelope. J. Bacteriol. 201:e00611-18. doi: 10.1128/JB.00611-18

DiGiuseppe, P. A., and Silhavy, T. J. (2003). Signal detection and target gene induction by the CpxRA two-component system. J. Bacteriol. 185, 2432-2440. doi: 10.1128/JB.185.8.2432-2440.2003

DiRita, V. J., Parsot, C., Jander, G., and Mekalanos, J. J. (1991). Regulatory cascade controls virulence in Vibrio cholerae. Proc. Natl. Acad. Sci. U. S. A. 88, 5403-5407. doi: 10.1073/pnas.88.12.5403

Douchin, V., Bohn, C., and Bouloc, P. (2006). Down-regulation of Porins by a Small RNA bypasses the essentiality of the regulated intramembrane proteolysis protease RseP in Escherichia coli. J. Biol. Chem. 281, 12253-12259. doi: 10.1074/jbc.M600819200

Drummond, N., Murphy, B. P., Ringwood, T., Prentice, M. B., Buckley, J. F. and Fanning, S. (2012). Yersinia enterocolitica: a brief review of the issues relating to the zoonotic pathogen, public health challenges, and the pork production chain. Foodborne Pathog. Dis. 9, 179-189. doi: 10.1089/fpd.201 1.0938

Du, H., Sheng, X., Zhang, H., Zou, X., Ni, B., Xu, S., et al. (2011). RpoE may promote flagellar gene expression in Salmonella enterica serovar typhi under hyperosmotic stress. Curr. Microbiol. 62, 492-500. doi: 10.1007/s00284-010-9734-y

Eriksson, S., Lucchini, S., Thompson, A., Rhen, M., and Hinton, J. C. D. (2003). Unravelling the biology of macrophage infection by gene expression profiling of intracellular Salmonella enterica. Mol. Microbiol. 47, 103-118. doi: 10.1046/j.1365-2958.2003.03313.x

Ernst, R. K., Guina, T., and Miller, S. I. (2001). Salmonella Typhimurium outer membrane remodeling: role in resistance to host innate immunity. Microbes Infect. 3, 1327-1334. doi: 10.1016/S1286-4579(01)01494-0

Evans, K. L., Kannan, S., Li, G., de Pedro, M. A., and Young, K. D. (2013). Eliminating a set of four penicillin binding proteins triggers the Rcs phosphorelay and Cpx stress responses in Escherichia coli. J. Bacteriol. 195, 4415-4424. doi: 10.1128/JB.00596-13

Fang, Ferric, C., Frawley, Elaine, R., Tapscott, T., and Vázquez-Torres, A. (2016). Bacterial stress responses during host infection. Cell Host Microbe 20, 133-143. doi: 10.1016/j.chom.2016.07.009

Fardini, Y., Chettab, K., Grepinet, O., Rochereau, S., Trotereau, J., Harvey, P., et al. (2007). The YfgL lipoprotein is essential for type III secretion system expression and virulence of Salmonella enterica serovar enteritidis. Infect. Immun. 75, 358-370. doi: 10.1128/IAI.00716-06

Fardini, Y., Trotereau, J., Bottreau, E., Souchard, C., Velge, P., and VirlogeuxPayant, I. (2009). Investigation of the role of the BAM complex and SurA chaperone in outer-membrane protein biogenesis and type III secretion system expression in Salmonella. Microbiology 155(Pt 5), 1613-1622. doi: 10.1099/mic.0.025155-0 
Field, M., Fromm, D., Al-Awqati, Q., and Greenough, W. B. III. (1972). Effect of cholera enterotoxin on ion transport across isolated ileal mucosa. J. Clin. Invest. 51, 796-804. doi: 10.1172/JCI106874

Figueroa-Bossi, N., Lemire, S., Maloriol, D., Balbontin, R., Casadesus, J., and Bossi, L. (2006). Loss of $\mathrm{Hfq}$ activates the sigmaE-dependent envelope stress response in Salmonella enterica. Mol. Microbiol. 62, 838-852. doi: 10.1111/j.1365-2958.2006.05413.x

Flores-Kim, J., and Darwin, A. J. (2012). Links between type III secretion and extracytoplasmic stress responses in Yersinia. Front. Cell. Infect. Microbiol. 2:125. doi: $10.3389 /$ fcimb.2012.00125

Flores-Kim, J., and Darwin, A. J. (2016). The phage shock protein response. Annu. Rev. Microbiol. 70, 83-101. doi: 10.1146/annurev-micro-102215-095359

Flynn, J. M., Levchenko, I., Sauer, R. T., and Baker, T. A. (2004). Modulating substrate choice: the SspB adaptor delivers a regulator of the extracytoplasmicstress response to the AAA+ protease ClpXP for degradation. Genes Dev. 18, 2292-2301. doi: 10.1101/gad.1240104

Friedrich, T., Dekovic, D. K., and Burschel, S. (2016). Assembly of the Escherichia coli NADH:ubiquinone oxidoreductase (respiratory complex I). Biochim. Biophys. Acta 1857, 214-223. doi: 10.1016/j.bbabio.2015.12.004

Frohlich, K. S., and Gottesman, S. (2018). Small regulatory RNAs in the enterobacterial response to envelope damage and oxidative stress. Microbiol. Spectr. 6. doi: 10.1128/microbiolspec.RWR-0022-2018

Fujimoto, M., Goto, R., Haneda, T., Okada, N., and Miki, T. (2018). Salmonella enterica serovar Typhimurium CpxRA two-component system contributes to gut colonization in salmonella-induced colitis. Infect. Immun. 86:e00280-18. doi: 10.1128/IAI.00280-18

Gallagher, L. A., and Manoil, C. (2001). Pseudomonas aeruginosa PAO1 Kills Caenorhabditis elegans by cyanide poisoning. J. Bacteriol. 183, 6207-6214. doi: 10.1128/JB.183.21.6207-6214.2001

Gal-Mor, O., and Segal, G. (2003). Identification of CpxR as a positive regulator of icm and dot virulence genes of Legionella pneumophila. J. Bacteriol. 185, 4908-4919. doi: 10.1128/JB.185.16.4908-4919.2003

Gangaiah, D., Raterman, E. L., Wu, H., Fortney, K. R., Gao, H., Liu, Y., et al. (2017). Both MisR (CpxR) and MisS (CpxA) are required for Neisseria gonorrhoeae infection in a murine model of lower genital tract infection. Infect. Immun. 85:e00307-17. doi: 10.1128/IAI.00307-17

Gangaiah, D., Zhang, X., Fortney, K. R., Baker, B., Liu, Y., Munson, R. S. Jr., et al. (2013). Activation of CpxRA in Haemophilus ducreyi primarily inhibits the expression of its targets, including major virulence determinants. J. Bacteriol. 195, 3486-3502. doi: 10.1128/JB.00372-13

Ge, X., Lyu, Z.-X., Liu, Y., Wang, R., Zhao, X.S., Fu, X., et al. (2014). Identification of FkpA as a key quality control factor for the biogenesis of outer membrane proteins under heat shock conditions. J. Bacteriol. 196, 672-680. doi: 10.1128/JB.01069-13

Gellatly, S. L., and Hancock, R. E. W. (2013). Pseudomonas aeruginosa: new insights into pathogenesis and host defenses. Pathog. Dis. 67, 159-173. doi: 10.1111/2049-632X.12033

Ghequire, M. G. K., Swings, T., Michiels, J., Buchanan, S. K., and De Mot, R. (2018). Hitting with a BAM selective killing by lectin-like bacteriocins. MBio 9:e02138-17. doi: 10.1128/mBio.02138-17

Giannakopoulou, N., Mendis, N., Zhu, L., Gruenheid, S., Faucher, S. P., and Le Moual, H. (2018). The virulence effect of CpxRA in Citrobacter rodentium is independent of the auxiliary proteins $\mathrm{NlpE}$ and CpxP. Front. Cell. Infect. Microbiol. 8:320. doi: 10.3389/fcimb.2018.00320

Gill,., D. M., and Meren, R. (1978). ADP-ribosylation of membrane proteins catalyzed by cholera toxin: Basis of the activation of adenylate cyclase. Proc. Natl. Acad. Sci. U. S. A. 75, 3050-3054. doi: 10.1073/pnas.75.7.3050

Gogol, E. B., Rhodius, V. A., Papenfort, K., Vogel, J., and Gross, C. A. (2011). Small RNAs endow a transcriptional activator with essential repressor functions for single-tier control of a global stress regulon. Proc. Natl. Acad. Sci. U. S. A. 108, 12875-12880. doi: 10.1073/pnas.1109379108

Govan, J. R., and Deretic, V. (1996). Microbial pathogenesis in cystic fibrosis: mucoid Pseudomonas aeruginosa and Burkholderia cepacia. Microbiol. Rev. 60, 539-574.

Grabenstein, J. P., Marceau, M., Pujol, C., Simonet, M., and Bliska, J. B. (2004). The response regulator PhoP of Yersinia pseudotuberculosis is important for replication in macrophages and for virulence. Infect. Immun. 72, 4973-4984. doi: 10.1128/IAI.72.9.4973-4984.2004
Grabowicz, M., Koren, D., and Silhavy, T. J. (2016). The CpxQ sRNA negatively regulates Skp to prevent mistargeting of beta-Barrel outer membrane proteins into the cytoplasmic membrane. MBio 7:e00312-16. doi: $10.1128 / \mathrm{mBio} .00312-16$

Grabowicz, M., and Silhavy, T. J. (2017). Redefining the essential trafficking pathway for outer membrane lipoproteins. Proc. Natl. Acad. Sci. U. S. A. 114, 4769-4774. doi: 10.1073/pnas.1702248114

Griffiths, G. L., Sigel, S. P., Payne, S. M., and Neilands, J. B. (1984). Vibriobactin, a siderophore from Vibrio cholerae. J. Biol. Chem. 259, 383-385.

Guest, R. L., Wang, J., Wong, J. L., and Raivio, T. L. (2017). A bacterial stress response regulates respiratory protein complexes to control envelope stress adaptation. J. Bacteriol. 199:e00153-17. doi: 10.1128/JB.00153-17

Guisbert, E., Rhodius, V. A., Ahuja, N., Witkin, E., and Gross, C. A. (2007). Hfq modulates the $\sigma \mathrm{E}$-mediated envelope stress response and the $\sigma 32$-mediated cytoplasmic stress response in Escherichia coli. J. Bacteriol. 189, 1963-1973. doi: 10.1128/JB.01243-06

Guo, M. S., Updegrove, T. B., Gogol, E. B., Shabalina, S. A., Gross, C. A., and Storz, G. (2014). MicL, a new $\sigma \mathrm{E}$-dependent sRNA, combats envelope stress by repressing synthesis of Lpp, the major outer membrane lipoprotein. Genes Dev. 28, 1620-1634. doi: 10.1101/gad.243485.114

Guo, X.-P., and Sun, Y.-C. (2017). New insights into the non-orthodox two component rcs phosphorelay system. Front. Microbiol. 8:2014. doi: $10.3389 /$ fmicb.2017.02014

Hagan, C. L., Westwood, D. B., and Kahne, D. (2013). bam lipoproteins assemble BamA in vitro. Biochemistry 52, 6108-6113. doi: 10.1021/bi400865z

Hagan, C. L., Wzorek, J. S., and Kahne, D. (2015). Inhibition of the $\beta$-barrel assembly machine by a peptide that binds BamD. Proc. Natl. Acad. Sci. U. S. A. 112, 2011-2016. doi: 10.1073/pnas.1415955112

Häse, C. C., and Mekalanos, J. J. (1998). TcpP protein is a positive regulator of virulence gene expression in Vibrio cholerae. Proc. Natl. Acad. Sci. U. S. A. 95, 730-734. doi: 10.1073/pnas.95.2.730

Hayden, J. D., and Ades, S. E. (2008). The extracytoplasmic stress factor, $\sigma \mathrm{E}$, is required to maintain cell envelope integrity in Escherichia coli. PLoS ONE 3:e1573. doi: 10.1371/journal.pone.0001573

Hernday, A. D., Braaten, B. A., Broitman-Maduro, G., Engelberts, P., and Low, D. A. (2004). Regulation of the pap epigenetic switch by CpxAR: phosphorylated CpxR inhibits transition to the phase ON state by competition with Lrp. Mol. Cell 16, 537-547. doi: 10.1016/j.molcel.2004.10.020

Heroven, A. K., Bohme, K., Rohde, M., and Dersch, P. (2008). A Csrtype regulatory system, including small non-coding RNAs, regulates the global virulence regulator RovA of Yersinia pseudotuberculosis through RovM. Mol. Microbiol. 68, 1179-1195. doi: 10.1111/j.1365-2958.2008.0 6218.x

Heroven, A. K., and Dersch, P. (2006). RovM, a novel LysR-type regulator of the virulence activator gene rovA, controls cell invasion, virulence and motility of Yersinia pseudotuberculosis. Mol. Microbiol. 62, 1469-1483. doi: 10.1111/j.1365-2958.2006.05458.x

Heusipp, G., Miller, V. L., and Schmidt, M. A. (2003). Identification of rpoE and nadB as host responsive elements of Yersinia enterocolitica. FEMS Microbiol. Lett. 226, 291-298. doi: 10.1016/S0378-1097(03)00613-X

Heusipp, G., Nelson, K. M., Schmidt, M. A., and Miller, V. L. (2004). Regulation of htrA expression in Yersinia enterocolitica. FEMS Microbiol. Lett. 231, 227-235. doi: 10.1016/S0378-1097(03)00962-5

Hirano, Y., Hossain, M. M., Takeda, K., Tokuda, H., and Miki, K. (2007). Structural studies of the Cpx pathway activator NlpE on the outer membrane of Escherichia coli. Structure 15, 963-976. doi: 10.1016/j.str.2007. 06.014

Holmqvist, E., and Wagner, E. G. H. (2017). Impact of bacterial sRNAs in stress responses. Biochem. Soc. Trans. 45, 1203-1212. doi: 10.1042/BST20 160363

Höltje, J. V., Mirelman, D., Sharon, N., and Schwarz, U. (1975). Novel type of murein transglycosylase in Escherichia coli. J. Bacteriol. 124, 1067-1076.

Hood, M. I., and Skaar, E. P. (2012). Nutritional immunity: transition metals at the pathogen-host interface. Nat. Rev. Microbiol. 10, 525-537. doi: $10.1038 /$ nrmicro2836

Hooda, Y., Shin, H. E., Bateman, T. J., and Moraes, T. F. (2017). Neisserial surface lipoproteins: structure, function and biogenesis. Pathog. Dis. 75:ftx010. doi: $10.1093 / \mathrm{femspd} / \mathrm{ft} \times 010$ 
Horne, S. M., Kottom, T. J., Nolan, L. K., and Young, K. D. (1997). Decreased intracellular survival of an fkpA mutant of Salmonella typhimurium Copenhagen. Infect. Immun. 65, 806-810.

Humphreys, S., Rowley, G., Stevenson, A., Anjum, M. F., Woodward, M. J., Gilbert, S., et al. (2004). Role of the two-component regulator CpxAR in the virulence of Salmonella enterica serotype Typhimurium. Infect. Immun. 72, 4654-4661. doi: 10.1128/IAI.72.8.4654-4661.2004

Humphreys, S., Rowley, G., Stevenson, A., Kenyon, W. J., Spector, M. P., and Roberts, M. (2003). Role of periplasmic peptidylprolyl isomerases in Salmonella enterica serovar Typhimurium virulence. Infect. Immun. 71, 5386-5388. doi: 10.1128/IAI.71.9.5386-5388.2003

Humphreys, S., Stevenson, A., Bacon, A., Weinhardt, A. B., and Roberts, M. (1999). The alternative sigma factor, $\sigma(\mathrm{E})$, is critically important for the virulence of Salmonella typhimurium. Infect. Immun. 67, 1560-1568.

Humphries, R. M., Griener, T. P., Vogt, S. L., Mulvey, G. L., Raivio, T., Donnenberg, M. S., et al. (2010). N-acetyllactosamine-induced retraction of bundle-forming pili regulates virulence-associated gene expression in enteropathogenic Escherichia coli. Mol. Microbiol. 76, 1111-1126. doi: 10.1111/j.1365-2958.2010.07192.x

Hung, D. L., Raivio, T. L., Jones, C. H., Silhavy, T. J., and Hultgren, S. J. (2001). Cpx signaling pathway monitors biogenesis and affects assembly and expression of P pili. EMBO J. 20, 1508-1518. doi: 10.1093/emboj/20.7.1508

Isaac, D. D., Pinkner, J. S., Hultgren, S. J., and Silhavy, T. J. (2005). The extracytoplasmic adaptor protein CpxP is degraded with substrate by DegP. Proc. Natl. Acad. Sci. U. S. A. 102, 17775-17779. doi: 10.1073/pnas.0508936102

Ito, K., and Inaba, K. (2008). The disulfide bond formation (Dsb) system. Curr. Opin. Struct. Biol. 18, 450-458. doi: 10.1016/j.sbi.2008.02.002

Jennings, E., Thurston, T. L. M., and Holden, D. W. (2017). Salmonella SPI-2 Type III secretion system effectors: molecular mechanisms and physiological consequences. Cell Host Microbe 22, 217-231. doi: 10.1016/j.chom.2017.07.009

Johansen, J., Rasmussen, A. A., Overgaard, M., and Valentin-Hansen, P. (2006). Conserved small non-coding RNAs that belong to the $\sigma \mathrm{E}$ regulon: role in down-regulation of outer membrane proteins. J. Mol. Biol. 364, 1-8. doi: 10.1016/j.jmb.2006.09.004

Jones, C. H., Danese, P. N., Pinkner, J. S., Silhavy, T. J., and Hultgren, S. J. (1997). The chaperone-assisted membrane release and folding pathway is sensed by two signal transduction systems. EMBO J. 16, 6394-6406. doi: 10.1093/emboj/16.21.6394

Kandler, J. L., Holley, C. L., Reimche, J. L., Dhulipala, V., Balthazar, J. T., Muszynski, A., et al. (2016). The MisR response regulator is necessary for intrinsic cationic antimicrobial peptide and aminoglycoside resistance in Neisseria gonorrhoeae. Antimicrob. Agents Chemother. 60, 4690-4700. doi: 10.1128/AAC.00823-16

Kanehara, K., Ito, K., and Akiyama, Y. (2002). YaeL (EcfE) activates the sigma(E) pathway of stress response through a site-2 cleavage of anti-sigma(E), RseA. Genes Dev. 16, 2147-2155. doi: 10.1101/gad.1002302

Kato, A., Hayashi, H., Nomura, W., Emori, H., Hagihara, K., and Utsumi, R. (2012). A connector-like factor, CacA, links RssB/RpoS and the CpxR/CpxA two-component system in Salmonella. BMC Microbiol. 12:224. doi: 10.1186/1471-2180-12-224

Kimkes, T. E. P., and Heinemann, M. (2018). Reassessing the role of the Escherichia coli CpxAR system in sensing surface contact. PLoS ONE 13:e0207181. doi: 10.1371/journal.pone.0207181

Kint, G., De Coster, D., Marchal, K., Vanderleyden, J., and De Keersmaecker, S. C. J. (2010). The small regulatory RNA molecule MicA is involved in Salmonella enterica serovar Typhimurium biofilm formation. BMC Microbiol. 10:276. doi: 10.1186/1471-2180-10-276

Klein, G., Kobylak, N., Lindner, B., Stupak, A., and Raina, S. (2014). Assembly of lipopolysaccharide in Escherichia coli requires the essential LapB heat shock protein. J. Biol. Chem. 289, 14829-14853. doi: 10.1074/jbc.M113.539494

Klein, G., and Raina, S. (2015). Regulated control of the assembly and diversity of LPS by noncoding sRNAs. Biomed Res. Int. 2015:153561. doi: $10.1155 / 2015 / 153561$

Klein, K., Sonnabend, M. S., Frank, L., Leibiger, K., Franz-Wachtel, M., Macek, B., et al. (2019). Deprivation of the periplasmic chaperone SurA reduces virulence and restores antibiotic susceptibility of multidrug-resistant Pseudomonas aeruginosa. Front. Microbiol. 10:100. doi: 10.3389/fmicb.2019.00100
Konovalova, A., Grabowicz, M., Balibar, C. J., Malinverni, J. C., Painter, R. E., Riley, D., et al. (2018). Inhibitor of intramembrane protease RseP blocks the $\sigma \mathrm{E}$ response causing lethal accumulation of unfolded outer membrane proteins. Proc. Natl. Acad. Sci. U. S. A. 115:E6614. doi: 10.1073/pnas.1806107115

Konovalova, A., Kahne, D. E., and Silhavy, T. J. (2017). Outer membrane biogenesis. Annu. Rev. Microbiol. 71, 539-556. doi: 10.1146/annurev-micro-090816-093754

Koronakis, V., Eswaran, J., and Hughes, C. (2004). Structure and function of TolC: the bacterial exit duct for proteins and drugs. Annu. Rev. Biochem. 73, 467-489. doi: 10.1146/annurev.biochem.73.011303.074104

Kovacikova, G., and Skorupski, K. (2002). The alternative sigma factor E plays an important role in intestinal survival and virulence in Vibrio cholerae. Infect. Immun. 70, 5355-5362. doi: 10.1128/IAI.70.10.5355-5362.2002

Kuhn, A., Koch, H. G., and Dalbey, R. E. (2017). Targeting and insertion of membrane proteins. EcoSal Plus 7, 1-27. doi: 10.1128/ecosalplus.ESP-0012-2016

Kunkle, D. E., Bina, X. R., and Bina, J. E. (2017). The Vibrio cholerae VexGH RND efflux system maintains cellular homeostasis by effluxing vibriobactin. MBio 8:e00126-17. doi: 10.1128/mBio.00126-17

Kwon, E., Kim, D. Y., Gross, C. A., Gross, J. D., and Kim, K. K. (2010). The crystal structure Escherichia coli Spy. Protein Sci. 19, 2252-2259. doi: 10.1002/pro.489

Labandeira-Rey, M., Brautigam, C. A., and Hansen, E. J. (2010). Characterization of the CpxRA regulon in Haemophilus ducreyi. Infect. Immun. 78, 4779-4791. doi: 10.1128/IAI.00678-10

Labandeira-Rey, M., Mock, J. R., and Hansen, E. J. (2009). Regulation of expression of the Haemophilus ducreyi LspB and LspA2 proteins by CpxR. Infect. Immun. 77, 3402-3411. doi: 10.1128/IAI.00292-09

Lasaro, M., Liu, Z., Bishar, R., Kelly, K., Chattopadhyay, S., Paul, S., et al. (2014). Escherichia coli isolate for studying colonization of the mouse intestine and its application to two-component signaling knockouts. J. Bacteriol. 196, 1723-1732. doi: 10.1128/JB.01296-13

LeClerc, G. J., Tartera, C., and Metcalf, E. S. (1998). Environmental regulation of Salmonella typhi invasion-defective mutants. Infect. Immun. 66, 682-691.

Lee, P. A., Tullman-Ercek, D., and Georgiou, G. (2006). The bacterial twin-arginine translocation pathway. Annu. Rev. Microbiol. 60, 373-395. doi: 10.1146/annurev.micro.60.080805.142212

Lee, Y. M., DiGiuseppe, P. A., Silhavy, T. J., and Hultgren, S. J. (2004). $\mathrm{P}$ pilus assembly motif necessary for activation of the CpxRA pathway by PapE in Escherichia coli. J. Bacteriol. 186, 4326-4337. doi: 10.1128/JB.186.13.4326-4337.2004

Lewis, C., Skovierova, H., Rowley, G., Rezuchova, B., Homerova, D., Stevenson, A., et al. (2008). Small outer-membrane lipoprotein, SmpA, is regulated by sigmaE and has a role in cell envelope integrity and virulence of Salmonella enterica serovar Typhimurium. Microbiology 154(Pt 3), 979-988. doi: 10.1099/mic.0.2007/011999-0

Li, J., Koh, J. J., Liu, S., Lakshminarayanan, R., Verma, C. S., and Beuerman, R. W. (2017). Membrane Active Antimicrobial Peptides: Translating Mechanistic Insights to Design. Front. Neurosci. 11, 73. doi: 10.3389/fnins.2017.00073

Li, J., Overall, C. C., Johnson, R. C., Jones, M. B., McDermott, J. E., Heffron, F., et al. (2015a). ChIP-Seq analysis of the sigmaE Regulon of Salmonella enterica serovar Typhimurium reveals new genes implicated in heat shock and oxidative stress response. PLoS ONE 10:e0138466. doi: 10.1371/journal.pone.0138466

Li, X., Wang, B., Feng, L., Kang, H., Qi, Y., Wang, J., et al. (2009). Cleavage of RseA by RseP requires a carboxyl-terminal hydrophobic amino acid following DegS cleavage. Proc. Natl. Acad. Sci. U. S. A. 106, 14837-14842. doi: 10.1073/pnas.0903289106

Li, Y., Hu, Y., Francis, M. S., and Chen, S. (2015b). RcsB positively regulates the Yersinia Ysc-Yop type III secretion system by activating expression of the master transcriptional regulator LcrF. Environ. Microbiol. 17, 1219-1233. doi: 10.1111/1462-2920.12556

Liu, J., Obi, I. R., Thanikkal, E. J., Kieselbach, T., and Francis, M. S. (2011). Phosphorylated CpxR restricts production of the RovA global regulator in Yersinia pseudotuberculosis. PLoS ONE 6:e23314. doi: 10.1371/journal.pone.0023314

Liu, J., Thanikkal, E. J., Obi, I. R., and Francis, M. S. (2012). Elevated CpxR P levels repress the Ysc-Yop type III secretion system of Yersinia pseudotuberculosis. Res. Microbiol. 163, 518-530. doi: 10.1016/j.resmic.2012.07.010 
Luirink, J., Yu, Z., Wagner, S., and de Gier, J. W. (2012). Biogenesis of inner membrane proteins in Escherichia coli. Biochim. Biophys. Acta 1817, 965-976. doi: 10.1016/j.bbabio.2011.12.006

Macritchie, D., and Raivio, T. (2009). Envelope stress responses. EcoSal Plus 3. doi: $10.1128 /$ ecosal.5.4.7

MacRitchie, D. M., Acosta, N., and Raivio, T. L. (2012). DegP is involved in Cpx-mediated posttranscriptional regulation of the type III secretion apparatus in enteropathogenic Escherichia coli. Infect. Immun. 80, 1766-1772. doi: 10.1128/IAI.05679-11

Macritchie, D. M., Ward, J. D., Nevesinjac, A. Z., and Raivio, T. L. (2008). Activation of the Cpx envelope stress response down-regulates expression of several locus of enterocyte effacement-encoded genes in enteropathogenic Escherichia coli. Infect. Immun. 76, 1465-1475. doi: 10.1128/IAI. 01265-07

Magnet, S., Dubost, L., Marie, A., Arthur, M., and Gutmann, L. (2008). Identification of the L,D-transpeptidases for peptidoglycan cross-linking in Escherichia coli. J. Bacteriol. 190, 4782-4785. doi: 10.1128/JB.00025-08

Mathee, K., Ciofu, O., Sternberg, C., Lindum, P. W., Campbell, J. I. A., Jensen, P., et al. (1999). Mucoid conversion of Pseudomonas aeruginosa by hydrogen peroxide: a mechanism for virulence activation in the cystic fibrosis lung. Microbiology 145, 1349-1357. doi: 10.1099/13500872-145-6-1349

Mathur, J., Davis, B. M., and Waldor, M. K. (2007). Antimicrobial peptides activate the Vibrio cholerae sigmaE regulon through an OmpU-dependent signalling pathway. Mol. Microbiol. 63, 848-858. doi: 10.1111/j.1365-2958.2006.05544.x

McCleary, W. R., and Stock, J. B. (1994). Acetyl phosphate and the activation of two-component response regulators. J. Biol. Chem. 269, 31567-31572.

McDaniel, T. K., Jarvis, K. G., Donnenberg, M. S., and Kaper, J. B. (1995). A genetic locus of enterocyte effacement conserved among diverse enterobacterial pathogens. Proc. Natl. Acad. Sci. U. S. A. 92, 1664-1668. doi: 10.1073/pnas.92.5.1664

McDaniel, T. K., and Kaper, J. B. (1997). A cloned pathogenecity island from enteropathogenic Escherichia coli confers the attaching and effacing phenotype on E. coli K-12. Mol. Microbiol. 23, 399-407. doi: 10.1046/j.1365-2958.1997.2311591.x

McGhie, E. J., Brawn, L. C., Hume, P. J., Humphreys, D., and Koronakis, V. (2009). Salmonella takes control: effector-driven manipulation of the host. Curr. Opin. Microbiol. 12, 117-124. doi: 10.1016/j.mib.2008.12.001

Mellies, J. L., Elliott, S. J., Sperandio, V., Donnenberg, M. S., and Kaper, J. B. (1999). The Per regulon of enteropathogenic Escherichia coli: identification of a regulatory cascade and a novel transcriptional activator, the locus of enterocyte effacement (LEE)-encoded regulator (Ler). Mol. Microbiol. 33, 296-306. doi: 10.1046/j.1365-2958.1999.01473.x

Misra, R., Stikeleather, R., and Gabriele, R. (2015). In vivo roles of BamA, $\mathrm{BamB}$ and $\mathrm{BamD}$ in the biogenesis of BamA, a core protein of the $\beta$ barrel assembly machine of Escherichia coli. J. Mol. Biol. 427, 1061-1074. doi: 10.1016/j.jmb.2014.04.021

Mitobe, J., Arakawa, E., and Watanabe, H. (2005). A sensor of the twocomponent system CpxA affects expression of the type III secretion system through posttranscriptional processing of InvE. J. Bacteriol. 187, 107-113. doi: $10.1128 /$ JB.187.1.107-113.2005

Miyadai, H., Tanaka-Masuda, K., Matsuyama, S., and Tokuda, H. (2004). Effects of lipoprotein overproduction on the induction of DegP $(\mathrm{HtrA})$ involved in quality control in the Escherichia coli periplasm. J. Biol. Chem. 279, 39807-39813. doi: 10.1074/jbc.M406390200

Morris, F. C., Wells, T. J., Bryant, J. A., Schager, A. E., Sevastsyanovich, Y. R., Squire, D. J. P., et al. (2018). YraP contributes to cell envelope integrity and virulence of Salmonella enterica serovar Typhimurium. Infect. Immun. 86, e00829-17. doi: 10.1128/IAI.00829-17

Muller, C., Bang, I. S., Velayudhan, J., Karlinsey, J., Papenfort, K., Vogel, J., et al. (2009). Acid stress activation of the sigma(E) stress response in Salmonella enterica serovar Typhimurium. Mol. Microbiol. 71, 1228-1238. doi: 10.1111/j.1365-2958.2009.06597.x

Nakayama, S., Kushiro, A., Asahara, T., Tanaka, R., Hu, L., Kopecko, D. J., et al. (2003). Activation of hilA expression at low $\mathrm{pH}$ requires the signal sensor CpxA, but not the cognate response regulator CpxR, in Salmonella enterica serovar Typhimurium. Microbiology 149(Pt 10), 2809-2817. doi: 10.1099/mic.0.26229-0
Nakayama, S., and Watanabe, H. (1995). Involvement of $c p x A$, a sensor of a two-component regulatory system, in the ph-dependent regulation of expression of Shigella sonnei virF Gene. J. Bacteriol. 177, 5062-5069. doi: $10.1128 /$ jb.177.17.5062-5069.1995

Nakayama, S., and Watanabe, H. (1998). Identification of $c p x R$ as a positive regulator essential for expression of the Shigella sonnei virF Gene. J. Bacteriol. $180,3522-3528$.

Nakayama, S., and Watanabe, H. (2006). Mechanism of hilA repression by 1,2-propanediol consists of two distinct pathways, one dependent on and the other independent of catabolic production of propionate, in Salmonella enterica serovar Typhimurium. J. Bacteriol. 188, 3121-3125. doi: $10.1128 / J B .188 .8 .3121-3125.2006$

Narita, S. I., and Tokuda, H. (2017). Bacterial lipoproteins; biogenesis, sorting and quality control. Biochim. Biophys. Acta 1862, 1414-1423. doi: 10.1016/j.bbalip.2016.11.009

Nevesinjac, A. Z., and Raivio, T. L. (2005). The Cpx envelope stress response affects expression of the type IV bundle-forming pili of enteropathogenic Escherichia coli. J. Bacteriol. 187, 672-686. doi: 10.1128/JB.187.2.672-686.2005

Obi, I. R., and Francis, M. S. (2013). Demarcating SurA activities required for outer membrane targeting of Yersinia pseudotuberculosis adhesins. Infect. Immun. 81, 2296-2308. doi: 10.1128/IAI.01208-12

Okkotsu, Y., Little, A. S., and Schurr, M. J. (2014). The Pseudomonas aeruginosa AlgZR two-component system coordinates multiple phenotypes. Front. Cell. Infect. Microbiol. 4:82. doi: 10.3389/fcimb.2014.00082

Okuda, S., and Tokuda, H. (2011). Lipoprotein sorting in bacteria. Annu. Rev. Microbiol. 65, 239-259. doi: 10.1146/annurev-micro-090110-102859

O'Loughlin, J. L., Spinner, J. L., Minnich, S. A., and Kobayashi, S. D. (2010). Yersinia pestis two-component gene regulatory systems promote survival in human neutrophils. Infect. Immun. 78, 773-782. doi: 10.1128/IAI.00718-09

Osborne, S. E., and Coombes, B. K. (2009). RpoE fine tunes expression of a subset of SsrB-regulated virulence factors in Salmonella enterica serovar Typhimurium. BMC Microbiol. 9:45. doi: 10.1186/14712180-9-45

Otto, K., and Silhavy, T. J. (2002). Surface sensing and adhesion of Escherichia coli controlled by the Cpx-signaling pathway. Proc. Natl. Acad. Sci. U. S. A. 99, 2287-2292. doi: 10.1073/pnas.042521699

Palmer, T., and Berks, B. C. (2012). The twin-arginine translocation (Tat) protein export pathway. Nat. Rev. Microbiol. 10, 483-496. doi: 10.1038/nrmicro2814

Pandey, S., Martins, K. L., and Mathee, K. (2016). "Posttranslational regulation of antisigma factors of RpoE: a comparison between the Escherichia coli and Pseudomonas aeruginosa systems," in Stress and Environmental Regulation of Gene Expression and Adaptation in Bacteria, ed F. J. de Brujin (Hoboken, NJ: John Wiley and Sons, Inc.), 361-367.

Papenfort, K., Pfeiffer, V., Mika, F., Lucchini, S., Hinton, J. C. D., and Vogel, J. (2006). SigmaE-dependent small RNAs of Salmonella respond to membrane stress by accelerating global omp mRNA decay. Mol. Microbiol. 62, 1674-1688. doi: 10.1111/j.1365-2958.2006.05524.x

Patel, G. J., and Kleinschmidt, J. H. (2013). The lipid bilayer-inserted membrane protein BamA of Escherichia coli facilitates insertion and folding of outer membrane protein A from its complex with Skp. Biochemistry 52, 3974-3986. doi: $10.1021 / b i 400103 t$

Pedersen, L. B., Murray, T., Popham, D. L., and Setlow, P. (1998). Characterization of $d a c C$, which encodes a new low-molecular-weight penicillin-binding protein in Bacillus subtilis. J. Bacteriol. 180, 4967-4973.

Petit-Härtlein, I., Rome, K., de Rosny, E., Molton, F., Duboc, C., Gueguen, E., et al. (2015). Biophysical and physiological characterization of ZraP from Escherichia coli the periplasmic accessory protein of the atypical ZraSR two-component system. Biochem. J. 472, 205-216. doi: 10.1042/BJ20150827

Plummer, A. M., and Fleming, K. G. (2016). From chaperones to the membrane with a BAM! Trends Biochem. Sci. 41, 872-882. doi: 10.1016/j.tibs.2016.06.005

Pogliano, J., Lynch, A. S., Belin, D., Lin, E. C. C., and Beckwith, J. (1997). Regulation of Escherichia coli cell envelope proteins involved in protein folding and degradation by the Cpx two-component system. Genes Dev. 11, 1169-1182. doi: 10.1101/gad.11.9.1169

Potvin, E., Sanschagrin, F., and Levesque, R. C. (2008). Sigma factors in Pseudomonas aeruginosa. FEMS Microbiol. Rev. 32, 38-55. doi: $10.1111 / j .1574-6976.2007 .00092 . x$ 
Price, N. L., and Raivio, T. L. (2009). Characterization of the Cpx regulon in Escherichia coli strain MC4100. J. Bacteriol. 191, 1798-1815. doi: 10.1128/JB.00798-08

Qiu, D., Eisinger, V. M., Head, N. E., Pier, G. B., and Yu, H. D. (2008). ClpXP proteases positively regulate alginate overexpression and mucoid conversion in Pseudomonas aeruginosa. Microbiology 154(Pt 7), 2119-2130. doi: $10.1099 /$ mic. $0.2008 / 017368-0$

Que, F., Wu, S., and Huang, R. (2013). Salmonella pathogenicity island 1(SPI-1) at work. Curr. Microbiol. 66, 582-587. doi: 10.1007/s00284-013-0307-8

Raffa, R. G., and Raivio, T. L. (2002). A third envelope stress signal transduction pathway in Escherichia coli. Mol. Microbiol. 45, 1599-1611. doi: $10.1046 /$ j.1365-2958.2002.03112.x

Raivio, T. L. (2014). Everything old is new again: an update on current research on the Cpx envelope stress response. Biochim. Biophys. Acta 1843, 1529-1541. doi: 10.1016/j.bbamcr.2013.10.018

Raivio, T. L., Laird, M. W., Joly, J. C., and Silhavy, T. J. (2000). Tethering of $\mathrm{CpxP}$ to the inner membrane prevents spheroplast induction of the Cpx envelope stress response. Mol. Microbiol. 37, 1186-1197. doi: 10.1046/j.1365-2958.2000.02074.x

Raivio, T. L., Leblanc, S. K., and Price, N. L. (2013). The Escherichia coli Cpx envelope stress response regulates genes of diverse function that impact antibiotic resistance and membrane integrity. J. Bacteriol. 195, 2755-2767. doi: 10.1128/JB.00105-13

Raivio, T. L., Popkin, D. L., and Silhavy, T. J. (1999). The Cpx envelope stress response is controlled by amplification and feedback inhibition. J. Bacteriol. $181,5263-5272$.

Raivio, T. L., and Silhavy, T. J. (1997). Transduction of envelope stress in Escherichia coli by the Cpx two-component system. J. Bacteriol. 179, 7724-7733. doi: 10.1128/jb.179.24.7724-7733.1997

Rao, J., Damron, F. H., Basler, M., Digiandomenico, A., Sherman, N. E., Fox, J. W., et al. (2011). Comparisons of two proteomic analyses of non-mucoid and mucoid Pseudomonas aeruginosa clinical isolates from a cystic fibrosis patient. Front. Microbiol. 2:162. doi: 10.3389/fmicb.2011.00162

Redford, P., Roesch, P. L., and Welch, R. A. (2003). degS is necessary for virulence and is among extraintestinal Escherichia coli genes induced in murine peritonitis. Infect. Immun. 71, 3088-3096. doi: 10.1128/IAI.71.6.3088-3096.2003

Redford, P., and Welch, R. A. (2006). Role of sigma E-regulated genes in Escherichia coli uropathogenesis. Infect. Immun. 74, 4030-4038. doi: 10.1128/IAI.01984-05

Rhodius, V. A., Suh, W. C., Nonaka, G., West, J., and Gross, C. A. (2006). Conserved and variable functions of the sigmaE stress response in related genomes. PLoS Biol. 4:e2. doi: 10.1371/journal.pbio.0040002

Rolhion, N., Carvalho, F. A., and Darfeuille-Michaud, A. (2007). OmpC and the sigma(E) regulatory pathway are involved in adhesion and invasion of the Crohn's disease-associated Escherichia coli strain LF82. Mol. Microbiol. 63, 1684-1700. doi: 10.1111/j.1365-2958.2007.05638.x

Ronnebaumer, K., Sander, G., Shutinoski, B., Schmidt, M. A., and Heusipp, G. (2009). Controlled activation of the Cpx system is essential for growth of Yersinia enterocolitica. FEMS Microbiol. Lett. 296, 274-281. doi: 10.1111/j.1574-6968.2009.01649.x

Rouvière, P. E., De Las Peñas, A., Mecsas, J., Lu, C. Z., Rudd, K. E., and Gross, C. A. (1995). rpoE, the gene encoding the second heatshock sigma factor, sigma E, in Escherichia coli. EMBO J. 14, 1032-1042. doi: 10.1002/j.1460-2075.1995.tb07084.x

Rowley, G., Skovierova, H., Stevenson, A., Rezuchova, B., Homerova, D., Lewis, C., et al. (2011). The periplasmic chaperone Skp is required for successful Salmonella Typhimurium infection in a murine typhoid model. Microbiology 157(Pt 3), 848-858. doi: 10.1099/mic.0.046011-0

Rowley, G., Spector, M., Kormanec, J., and Roberts, M. (2006). Pushing the envelope: extracytoplasmic stress responses in bacterial pathogens. Nat. Rev. Microbiol. 4, 383-394. doi: 10.1038/nrmicrol394

Rowley, G., Stevenson, A., Kormanec, J., and Roberts, M. (2005). Effect of inactivation of degS on Salmonella enterica serovar typhimurium in vitro and in vivo. Infect. Immun. 73, 459-463. doi: 10.1128/IAI.73.1.459-463.2005

Ruiz, N., Kahne, D., and Silhavy, T. J. (2006). Advances in understanding bacterial outer-membrane biogenesis. Nat. Rev. Microbiol. 4, 57-66. doi: 10.1038/nrmicro1322
Shimizu, T., Ichimura, K., and Noda, M. (2016). The surface sensor NlpE of enterohemorrhagic Escherichia coli contributes to regulation of the type III secretion system and flagella by the Cpx response to adhesion. Infect. Immun. 84, 537-549. doi: 10.1128/IAI.00881-15

Shimohata, N., Chiba, S., Saikawa, N., Ito, K., and Akiyama, Y. (2002). The Cpx stress response system of Escherichia coli senses plasma membrane proteins and controls HtpX, a membrane protease with a cytosolic active site. Genes Cells 7, 653-662. doi: 10.1046/j.1365-2443.2002.00554.x

Silhavy, T. J., Kahne, D., and Walker, S. (2010). The bacterial cell envelope. Cold Spring Harb. Perspect. Biol. 2, a000414. doi: 10.1101/cshperspect.a000414

Siroy, A., Cosette, P., Seyer, D., Lemaître-Guillier, C., Vallenet, D., Van Dorsselaer, A., et al. (2006). Global comparison of the membrane subproteomes between a multidrug-resistant Acinetobacter baumanii strain and a reference strain. J. Proteome Res. 5, 3385-3398. doi: 10.1021/pr060372s

Sittka, A., Pfeiffer, V., Tedin, K., and Vogel, J. (2007). The RNA chaperone Hfq is essential for the virulence of Salmonella Typhimurium. Mol. Microbiol. 63, 193-217. doi: 10.1111/j.1365-2958.2006.05489.x

Sklar, J. G., Wu, T., Gronenberg, L. S., Malinverni, J. C., Kahne, D., and Silhavy, T. J. (2007a). Lipoprotein SmpA is a component of the YaeT complex that assembles outer membrane proteins in Escherichia coli. Proc. Natl. Acad. Sci. U. S. A. 104, 6400-6405. doi: 10.1073/pnas.0701579104

Sklar, J. G., Wu, T., Kahne, D., and Silhavy, T. J. (2007b). Defining the roles of the periplasmic chaperones SurA, Skp, and DegP in Escherichia coli. Genes Dev. 21, 2473-2484. doi: 10.1101/gad.1581007

Skovierova, H., Rowley, G., Rezuchova, B., Homerova, D., Lewis, C., Roberts, M., et al. (2006). Identification of the sigmaE regulon of Salmonella enterica serovar Typhimurium. Microbiology 152(Pt 5), 1347-1359. doi: 10.1099/mic.0.2 8744-0

Slamti, L., and Waldor, M. K. (2009). Genetic analysis of activation of the Vibrio cholerae Cpx pathway. J. Bacteriol. 191, 5044-5056. doi: 10.1128/JB.00406-09

Snyder, W. B., Davis, L. J. B., Danese, P. N., Cosma, C. L., and Silhavy, T. J. (1995). Overproduction of NlpE, a new outer membrane lipoprotein suppresses the toxicity of periplasmic LacZ by activation of the Cpx signal transduction pathway. J. Bacteriol. 177, 4216-4223. doi: 10.1128/jb.177.15.4216-4223.1995

Sohn, J., Grant, R. A., and Sauer, R. T. (2009). OMP peptides activate the DegS stress-sensor protease by a relief of inhibition mechanism. Structure 17, 1411-1421. doi: 10.1016/j.str.2009.07.017

Song, T., Sabharwal, D., Gurung, J. M., Cheng, A. T., Sjöström, A. E., Yildiz, F. H., et al. (2014). Vibrio cholerae utilizes direct sRNA regulation in expression of a biofilm matrix protein. PLoS ONE 9:e101280. doi: 10.1371 /journal.pone. 0101280

Song, T., and Wai, S. N. (2009). A novel sRNA that modulates virulence and environmental fitness of Vibrio cholerae. RNA Biol. 6, 254-258. doi: 10.4161/rna.6.3.8371

Southern, S. J., Scott, A. E., Jenner, D. C., Ireland, P. M., Norville, I. H., and SarkarTyson, M. (2016). Survival protein A is essential for virulence in Yersinia pestis. Microb. Pathog. 92, 50-53. doi: 10.1016/j.micpath.2015.12.013

Spiess, C., Beil, A., and Ehrmann, M. (1999). A temperature-dependent switch from chaperone to protease in a widely conserved heat shock protein. Cell 97, 339-347. doi: 10.1016/S0092-8674(00)80743-6

Spöring, I., Felgner, S., Preuße, M., Eckweiler, D., Rohde, M., Häussler, S., et al. (2018). Regulation of flagellum biosynthesis in response to cell envelope stress in Salmonella enterica serovar Typhimurium. MBio 9:e0073617. doi: $10.1128 / \mathrm{mBio} .00736-17$

Srikumar, S., Kröger, C., Hébrard, M., Colgan, A., Owen, S. V., Sivasankaran, S. K., et al. (2015). RNA-seq brings new insights to the intra-macrophage transcriptome of Salmonella Typhimurium. PLoS Pathog. 11:e1005262. doi: 10.1371/journal.ppat.1005262

Stankeviciute, G., Miguel, A. V., Radkov, A., Chou, S., Huang, K. C., and Klein, E. A. (2019). Differential modes of crosslinking establish spatially distinct regions of peptidoglycan in Caulobacter crescentus. Mol. Microbiol. 111, 995-1008. doi: $10.1111 / \mathrm{mmi} .14199$

Storek, K. M., Auerbach, M. R., Shi, H., Garcia, N. K., Sun, D., Nickerson, N. N., et al. (2018). Monoclonal antibody targeting the $\beta$-barrel assembly machine of Escherichia coli is bactericidal. Proc. Natl. Acad. Sci. U. S. A. 115, 3692-3697. doi: 10.1073/pnas. 1800043115

Subramaniam, S., Muller, V. S., Hering, N. A., Mollenkopf, H., Becker, D., Heroven, A. K., et al. (2019). Contribution of the Cpx envelope stress 
system to metabolism and virulence regulation in Salmonella enterica serovar Typhimurium. PLoS ONE 14:e0211584. doi: 10.1371/journal.pone.0211584

Sydenham, M., Douce, G., Bowe, F., Ahmed, S., Chatfield, S., and Dougan, G. (2000). Salmonella enterica serovar Typhimurium surA mutants are attenuated and effective live oral vaccines. Infect. Immun. 68, 1109-1115. doi: 10.1128/IAI.68.3.1109-1115.2000

Szewczyk, J., and Collet, J. F. (2016). The journey of lipoproteins through the cell: one birthplace, multiple destinations. Adv. Microb. Physiol. 69, 1-50. doi: $10.1016 /$ bs.ampbs.2016.07.003

Tanaka, K., Matsuyama, S. I., and Tokuda, H. (2001). Deletion of lolB, encoding an outer membrane lipoprotein, is lethal for Escherichia coli and causes accumulation of lipoprotein localization intermediates in the periplasm. $J$. Bacteriol. 183, 6538-6542. doi: 10.1128/JB.183.22.6538-6542.2001

Taylor, D. L., Bina, X. R., and Bina, J. E. (2012). Vibrio cholerae VexH encodes a multiple drug efflux pump that contributes to the production of cholera toxin and the toxin co-regulated pilus. PLOS ONE 7:e38208. doi: 10.1371/journal.pone.0038208

Taylor, D. L., Bina, X. R., Slamti, L., Waldor, M. K., and Bina, J. E. (2014). Reciprocal regulation of resistance-nodulation-division efflux systems and the Cpx two-component system in Vibrio cholerae. Infect. Immun. 82, 2980-2991. doi: 10.1128/IAI.00025-14

Testerman, T. L., Vazquez-Torres, A., Xu, Y., Jones-Carson, J., Libby, S. J., and Fang, F. C. (2002). The alternative sigma factor sigmaE controls antioxidant defences required for Salmonella virulence and stationary-phase survival. Mol. Microbiol. 43, 771-782. doi: 10.1046/j.1365-2958.2002.02787.x

Thanikkal, E. J., Gahlot, D. K., Liu, J., Fredriksson Sundbom, M., Gurung, J. M., Ruuth, K., et al. (2019). The Yersinia pseudotuberculosis Cpx envelope stress system contributes to transcriptional activation of rovM. Virulence 10, 37-57. doi: 10.1080/21505594.2018.1556151

Thomassin, J. L., Giannakopoulou, N., Zhu, L., Gross, J., Salmon, K., Leclerc, J. M., et al. (2015). The CpxRA two-component system is essential for Citrobacter rodentium virulence. Infect. Immun. 83, 1919-1928. doi: 10.1128/IAI.00194-15

Thomassin, J. L., Leclerc, J. M., Giannakopoulou, N., Zhu, L., Salmon, K., Portt, A., et al. (2017). Systematic analysis of two-component systems in Citrobacter rodentium reveals positive and negative roles in virulence. Infect. Immun. 85:e00654-16. doi: 10.1128/IAI.00654-16

Tobe, T., and Sasakawa, C. (2001). Role of bundle-forming pilus of enteropathogenic Escherichia coli in host cell adherence and in microcolony development. Cell. Microbiol. 3, 579-585. doi: 10.1046/j.1462-5822.2001.00136.x

Tschauner, K., Hornschemeyer, P., Muller, V. S., and Hunke, S. (2014). Dynamic interaction between the CpxA sensor kinase and the periplasmic accessory protein CpxP mediates signal recognition in E. coli. PLoS ONE 9:e107383. doi: 10.1371/journal.pone.0107383

Tsirigotaki, A., De Geyter, J., Sostaric, N., Economou, A., and Karamanou, S. (2017). Protein export through the bacterial Sec pathway. Nat. Rev. Microbiol. 15, 21-36. doi: 10.1038/nrmicro.2016.161

Tzeng, Y. L., Datta, A., Ambrose, K., Lo, M., Davies, J. K., Carlson, R. W., et al. (2004). The MisR/MisS two-component regulatory system influences inner core structure and immunotype of lipooligosaccharide in Neisseria meningitidis. J. Biol. Chem. 279, 35053-35062. doi: 10.1074/jbc.M401433200

Tzeng, Y. L., Kahler, C. M., Zhang, X., and Stephens, D. S. (2008). MisR/MisS two-component regulon in Neisseria meningitidis. Infect. Immun. 76, 704-716. doi: 10.1128/IAI.01007-07

Ünal, C. M., and Steinert, M. (2014). Microbial Peptidyl-Prolyl cis/trans Isomerases (PPIases): virulence factors and potential alternative drug targets. Microbiol. Mol. Biol. Rev. 78, 544-571. doi: 10.1128/MMBR.00015-14

Vincent, C. D., Buscher, B. A., Friedman, J. R., Williams, L. A., Bardill, P., and Vogel, J. P. (2006). Identification of non-dot/icm suppressors of the Legionella pneumophila deltadotL lethality phenotype. J. Bacteriol. 188, 8231-8243. doi: 10.1128/JB.00937-06

Vlahou, G., Schmidt, O., Wagner, B., Uenlue, H., Dersch, P., Rivero, F., et al. (2009). Yersinia outer protein YopE affects the actin cytoskeleton in Dictyostelium discoideum through targeting of multiple Rho family GTPases. BMC Microbiol. 9:138. doi: 10.1186/1471-2180-9-138

Vogel, J., and Papenfort, K. (2006). Small non-coding RNAs and the bacterial outer membrane. Curr. Opin. Microbiol. 9, 605-611. doi: 10.1016/j.mib.2006. 10.006
Vogt, S. L., Evans, A. D., Guest, R. L., and Raivio, T. L. (2014). The Cpx envelope stress response regulates and is regulated by small noncoding RNAs. J. Bacteriol. 196, 4229-4238. doi: 10.1128/JB.02138-14

Vogt, S. L., Nevesinjac, A. Z., Humphries, R. M., Donnenberg, M. S., Armstrong, G. D., and Raivio, T. L. (2010). The Cpx envelope stress response both facilitates and inhibits elaboration of the enteropathogenic Escherichia coli bundle-forming pilus. Mol. Microbiol. 76, 1095-1110. doi: 10.1111/j.1365-2958.2010.07145.x

Vogt, S. L., and Raivio, T. L. (2012). Just scratching the surface: an expanding view of the Cpx envelope stress response. FEMS Microbiol. Lett. 326, 2-11. doi: 10.1111/j.1574-6968.2011.02406.x

Vogt, S. L., Scholz, R., Peng, Y., Guest, R. L., Scott, N. E., Woodward, S. E., et al. (2019). Characterization of the Citrobacter rodentium Cpx regulon and its role in host infection. Mol. Microbiol. 111, 700-716. doi: 10.1111/mmi.14182

Voulhoux, R., Bos, M. P., Geurtsen, J., Mols, M., and Tommassen, J. (2003). Role of a highly conserved bacterial protein in outer membrane protein assembly. Science 299, 262-265. doi: 10.1126/science.1078973

Vuong, P., Bennion, D., Mantei, J., Frost, D., and Misra, R. (2008). Analysis of YfgL and YaeT interactions through bioinformatics, mutagenesis, and biochemistry. J. Bacteriol. 190:1507. doi: 10.1128/JB.01477-07

Wall, E., Majdalani, N., and Gottesman, S. (2018). The complex Rcs regulatory cascade. Annu. Rev. Microbiol. 72, 111-139. doi: 10.1146/annurev-micro-090817-062640

Walsh, N. P., Alba, B. M., Bose, B., Gross, C. A., and Sauer, R. T. (2003). OMP peptide signals initiate the envelope-stress response by activating DegS protease via relief of inhibition mediated by its PDZ domain. Cell 113, 61-71. doi: 10.1016/S0092-8674(03)00203-4

Walsham, A. D., MacKenzie, D. A., Cook, V., Wemyss-Holden, S., Hews, C. L., Juge, N., et al. (2016). Lactobacillus reuteri inhibition of enteropathogenic Escherichia coli adherence to human intestinal epithelium. Front. Microbiol. 7:244. doi: $10.3389 /$ fmicb.2016.00244

Weatherspoon-Griffin, N., Zhao, G., Kong, W., Kong, Y., Morigen, AndrewsPolymenis, H., et al. (2011). The CpxR/CpxA two-component system up-regulates two Tat-dependent peptidoglycan amidases to confer bacterial resistance to antimicrobial peptide. J. Biol. Chem. 286, 5529-5539. doi: $10.1074 /$ jbc.M110.200352

Weirich, J., Brautigam, C., Muhlenkamp, M., Franz-Wachtel, M., Macek, B., Meuskens, I., et al. (2017). Identifying components required for OMP biogenesis as novel targets for antiinfective drugs. Virulence 8, 1170-1188. doi: 10.1080/21505594.2016.1278333

Wolfe, A. J., Parikh, N., Lima, B. P., and Zemaitaitis, B. (2008). Signal integration by the two-component signal transduction response regulator CpxR. J. Bacteriol. 190, 2314-2322. doi: 10.1128/JB.01906-07

Wullt, B., Bergsten, G., Connell, H., Röllano, P., Gebretsadik, N., Hull, R., et al. (2000). P fimbriae enhance the early establishment of Escherichia col in the human urinary tract. Mol. Microbiol. 38, 456-464. doi: 10.1046/j.1365-2958.2000.02165.x

Xie, X., Zhang, H., Zheng, Y., Li, A., Wang, M., Zhou, H., et al. (2016). RpoE is a putative antibiotic resistance regulator of Salmonella enteric serovar Typhi. Curr. Microbiol. 72, 457-464. doi: 10.1007/s00284-015-0983-7

Yakhnina, A. A., McManus, H. R., and Bernhardt, T. G. (2015). The cell wall amidase AmiB is essential for Pseudomonas aeruginosa cell division, drug resistance and viability. Mol. Microbiol. 97, 957-973. doi: 10.1111/mmi.13077

Yamamoto, K., and Ishihama, A. (2006). Characterization of copper-inducible promoters regulated by $\mathrm{CpxA} / \mathrm{CpxR}$ in Escherichia coli. Biosci. Biotechnol. Biochem. 70, 1688-1695. doi: 10.1271/bbb.60024

Yamamoto, T., Sashinami, H., Takaya, A., Tomoyasu, T., Matsui, H., Kikuchi, Y., et al. (2001). Disruption of the genes for ClpXP protease in Salmonella enterica serovar Typhimurium results in persistent infection in mice, and development of persistence requires endogenous gamma interferon and tumor necrosis factor alpha. Infect. Immun. 69, 3164-3174. doi: 10.1128/IAI.69.5.3164-3174.2001

Yang, Y., Liao, Y., Ma, Y., Gong, W., and Zhu, G. (2017). The role of major virulence factors of AIEC involved in inflammatory bowl disease-a mini-review. Appl. Microbiol. Biotechnol. 101, 7781-7787. doi: 10.1007/s00253-017-8507-y

Yoon, H., McDermott, J. E., Porwollik, S., McClelland, M., and Heffron, F. (2009). Coordinated regulation of virulence during systemic infection 
of Salmonella enterica serovar Typhimurium. PLoS Pathog. 5:e1000306. doi: 10.1371/journal.ppat.1000306

Yu, H., Boucher, J. C., Hibler, N. S., and Deretic, V. (1996). Virulence properties of Pseudomonas aeruginosa lacking the extreme-stress sigma factor AlgU (sigmaE). Infect. Immun. 64, 2774-2781.

Yu, H., Schurr, M. J., and Deretic, V. (1995). Functional equivalence of Escherichia coli sigma E and Pseudomonas aeruginosa AlgU: E. coli rpoE restores mucoidy and reduces sensitivity to reactive oxygen intermediates in algU mutants of $P$. aeruginosa. J. Bacteriol. 177, 3259-3268. doi: 10.1128/jb.177.11.3259-3268.1995

Zhang, H., Jia, Y., Xie, X., Wang, M., Zheng, Y., Xu, S., et al. (2016). RpoE promotes invasion and intracellular survival by regulating SPI-1 and SPI- 2 in Salmonella enterica serovar Typhi. Fut. Microbiol. 11, 1011-1024. doi: 10.2217/fmb.16.19

Zhou, X., Keller, R., Volkmer, R., Krauss, N., Scheerer, P., and Hunke, S. (2011). Structural basis for two-component system inhibition and pilus sensing by the auxiliary CpxP protein. J. Biol. Chem. 286, 9805-9814. doi: 10.1074/jbc.M110.194092
Zhu, J., Miller, M. B., Vance, R. E., Dziejman, M., Bassler, B. L., and Mekalanos, J. J. (2002). Quorum-sensing regulators control virulence gene expression in Vibrio cholerae. Proc. Natl. Acad. Sci. U. S. A. 99, 3129-3134. doi: 10.1073/pnas.052694299

Conflict of Interest Statement: The authors declare that the research was conducted in the absence of any commercial or financial relationships that could be construed as a potential conflict of interest.

Copyright (c) 2019 Hews, Cho, Rowley and Raivio. This is an open-access article distributed under the terms of the Creative Commons Attribution License (CC BY). The use, distribution or reproduction in other forums is permitted, provided the original author(s) and the copyright owner(s) are credited and that the original publication in this journal is cited, in accordance with accepted academic practice. No use, distribution or reproduction is permitted which does not comply with these terms. 